\title{
Rhythmic coordination of hippocampal-prefrontal ensembles for odor-place associative memory and decision making
}

Authors: Claire A. Symanski ${ }^{1}$, John H. Bladon², Emi T. Kullberg² and Shantanu P. Jadhav'1,2,3*

\author{
Affiliations: \\ ${ }^{1}$ Graduate Program in Neuroscience, Brandeis University, Waltham, MA, 02453, USA \\ ${ }^{2}$ Neuroscience Program, Department of Psychology, Volen National Center for Complex \\ Systems, Brandeis University, Waltham, MA, 02453, USA \\ ${ }^{3}$ Lead Contact \\ ${ }^{*}$ Correspondence to: shantanu@brandeis.edu (S.P.J.)
}




\section{ABSTRACT}

Memory-guided decision making involves long-range coordination across sensory and

3 cognitive brain networks, with key roles for the hippocampus and prefrontal cortex (PFC). To

4 investigate coordination mechanisms that mediate sensory-cued decision making based on

5 learned associations, we monitored activity in hippocampus (CA1), PFC, and olfactory bulb in

6 rats performing an odor-place associative memory and decision task on a T-maze. During odor

7 sampling, the beta $(15-30 \mathrm{~Hz})$ and respiratory $(7-8 \mathrm{~Hz})$ rhythms (RR) were prominent and

8 coherent across the three regions, with beta specifically linked to fast decisions. Single neurons

9 and ensembles in both CA1 and PFC encoded and predicted animals' upcoming choices, with

10 choice-encoding dynamics linked specifically with beta rhythm coordination. Beta phase

11 modulation of CA1 interneuron activity was linked to accurate decisions, suggesting a

12 mechanism for enabling temporal coordination. Our findings indicate that beta rhythm

13 coordination within the hippocampal-prefrontal network supports utilization of odor cues for

14 memory-guided decision making.

\section{KEYWORDS}

16 Hippocampus; Prefrontal cortex; Beta oscillations; Respiratory rhythm; Phase locking; Decision 17 making; Olfactory Bulb 


\section{INTRODUCTION}

The ability to recall associations from memory and use them to guide behavior is a key aspect of cognition across species. Animals can associate sensory cues in the environment with rewarding and noxious experiences and utilize these cues for adaptive behavior. Memoryguided decision making demonstrates the brain's remarkable ability to link familiar cues with actions and beneficial outcomes, but little is known about the mechanisms responsible for this cognitive function.

Neurons that encode learned associations and reflect upcoming choice behavior have been reported in multiple regions in different sensory modalities ${ }^{1-14}$. However, sensory cued decision-making based on learned associations necessarily involves a brain-wide network that links primary sensory areas, the medial temporal lobe, and higher cortical areas involved in executive function. Numerous studies have highlighted the significance of the hippocampus and prefrontal cortex (PFC) in cognitive processing related to memory and decision making ${ }^{15-19}$. Both regions are known to encode behaviorally relevant cues and task features ${ }^{2-4,20-22}$, and have been shown to play key roles in memory recall ${ }^{23-26}$. Notably, coordinated activity between the hippocampus and PFC, supported by bidirectional anatomical connections ${ }^{27-29}$, has been shown to be critical for learning and memory-guided behavior ${ }^{30-35}$. Therefore, we focused on the coordinated interactions between hippocampus and PFC as a potential key mechanism through which learned associations are recalled and translated into memory-guided decisions.

Several studies have shown that rhythmic network oscillations in the local field potential (LFP) are involved in long-range interactions between the hippocampus and PFC $30,36-40$. In particular, phase coherence in distinct frequency bands across this network has been suggested as a mechanism for network coordination underlying mnemonic functions. Notably, hippocampal-prefrontal coherence in the theta rhythm $(6-12 \mathrm{~Hz})$ plays a role in spatial working memory and acquisition of spatial tasks $37,38,41,42$. However, whether similar mechanisms of 
coordination between hippocampus and PFC underlie decision making based on sensory cued associations is unclear.

Rodents rely heavily on odor cues for navigation and foraging, so odor memories are highly salient and robust ${ }^{43-46}$, making olfactory memory tasks ideally suited for studying memoryguided decision making. Previous studies using odor memory tasks have found prominent beta $(15-30 \mathrm{~Hz})$ oscillations in olfactory regions and the medial temporal lobe during cue sampling, suggesting that the beta rhythm acts as a potential mode of long-range communication for olfactory information processing ${ }^{5,47-50}$. In addition to the beta rhythm, the respiratory rhythm (RR; 7-8 Hz), driven by the animal's breathing cycle, is also prominent in the hippocampus during mnemonic processing of odor stimuli ${ }^{51-55}$. However, not much is known about the roles of these rhythms in coordinating activity in the hippocampal-prefrontal network during odor-cued decision making.

To elucidate these mechanisms, we employed an odor-place association task in which rats were required to choose the correct trajectory on a T-maze by recalling and utilizing familiar associations between odor cues and reward locations. While rats were performing the task, we recorded simultaneously from the hippocampus and PFC. Given the involvement of both of these regions in odor-memory tasks ${ }^{34,56-62}$, and the role of hippocampal-prefrontal networks in memory-guided behavior ${ }^{23,24,26,63-65}$, we hypothesized that rhythmic activity in this network may govern the cellular representation of odor-cued decisions underlying behavioral choices. We also monitored LFP activity in the olfactory bulb $(\mathrm{OB})$ in addition to CA1 and PFC, for robust samplings of previously reported olfactory rhythms such as beta and $R R$, which have been

64 implicated in cognitive processing of olfactory stimuli66,67. Our results point to a specific role of 65 beta rhythms in coordinating the olfactory-hippocampal-prefrontal network for utilizing learned odor-place associations to inform decisions, and shed light on the cellular and network mechanisms underlying this process. 


\section{RESULTS}

Odor-place associative memory and decision-making task

The odor-cued spatial associative memory task required rats to sniff at an odor port

72

73

74

where one of two possible odors was presented using a calibrated olfactometer (see Methods). The rats were required to choose the correct associated reward arm on the T-maze based on the sampled odor identity, where they would receive a reward of evaporated milk (Fig. 1a).

Rats were habituated to the maze and pre-trained on the task before surgical implant of a tetrode microdrive array for recording neural data. Following post-operative recovery and during electrophysiological recording, animals maintained a high level of performance on the task, indicating accurate decision making based on cued recall of odor-place associations (Fig. 1b, $n=8$ rats, $77.0 \% \pm 1.3 \%$, mean \pm s.e.m.). Rats were required to hold their nose in the odor port for a minimum of 0.50 seconds on each trial, but could continue sniffing the odor for any length of time after the minimum threshold was reached. The odor was continuously dispensed for the entire duration of time that the rat held its nose in the odor port and was only turned off once the rat disengaged from the odor-port. The average odor-sampling duration before odor port disengagement and odor offset was $0.82 \pm 0.02$ seconds (mean \pm s.e.m. across sessions), and this duration was similar between correct and incorrect trials (Fig. 1c; signed-rank test, $p=$ 0.71, distribution for all trials shown in Supplementary Fig. 1a). The animals' average velocity during the task showed a decrease in speed from the pre-odor period to the odor sampling period in the odor port, followed by an increase in speed after they left the odor port to run to the reward location (Supplementary Fig. 1b-c). We observed rapid movement away from the odorport only after odor-port disengagement (Supplementary Fig. 1d). In two animals, a thermocouple was implanted in the nasal cavity to measure the sniff rhythm (see Methods). There was a small but significant increase in sniff rate during the odor sampling period $(7.1 \pm$ 

s.e.m.) (Supplementary Fig. 1e). direction away from the odor port matched the direction of the T-maze reward arm that they would ultimately choose on that trial. This behavioral phenomenon was not required for successful performance of the task, and it occurred regardless of whether the trial was correct or incorrect (Fig. 1d; binomial tests, correct trials: $p=0$; incorrect trials: $p=1.4 \mathrm{e}-108$ ). This observation indicates that the rats recall the odor-place association and choose the reward location for each trial during the odor sampling period, before exiting the odor port to run toward to odor-cued recall of the learned association and priming of the subsequent decision to turn toward the reward location, with behavioral report of the decision occurring at odor port offset. We therefore termed this odor sampling period as "the decision-making period," since it provides a temporal window between odor onset and odor port exit to investigate mechanisms underlying odor-cued decision making.

\section{Beta rhythms support decision making based on odor-place associations}

112 network dynamics that underlie coordination of brain regions during this period. We used a

113 tetrode microdrive array to record local field potentials (LFPs) and single units from the dorsal

114 CA1 region of the hippocampus, the prelimbic region of the prefrontal cortex (PFC, primarily

115 prelimbic area), and the olfactory bulb (OB, only LFP) in rats as they performed the odor-place

116 association task (see Methods; Supplementary Fig. 2a-b). The thermocouple signal and LFP 
traces from CA1, PFC, and OB from an example trial are shown in Fig. 2a, along with the same LFP signals filtered in the $15-30 \mathrm{~Hz}$ band (additional example shown in Supplementary Fig. 2c).

We observed a strong increase in power in the beta band $(15-30 \mathrm{~Hz})$ during this decision-making period compared to a time-matched pre-odor period across all three regions tasks have been reported previously in OB, CA1, and lateral entorhinal cortex ${ }^{5,47-49}$. The respiratory rhythm $(\mathrm{RR}, 7-8 \mathrm{~Hz})$ was also prominent in the LFP in all three regions ${ }^{51,53}$, showing an increase in power in PFC and OB during odor-sampling compared to time-matched pre-odor periods (Fig. 2b; Supplementary Fig. 2d,f). This rhythm, which corresponds to the respiration rate during odor sampling, has previously been shown to be physiologically and mechanistically distinct from the $6-12 \mathrm{~Hz}$ hippocampal theta rhythm ${ }^{51,53}$, although there is overlap between the two frequency bands. Following odor port disengagement and the initiation of running down the track, we observed a small shift in the dominant LFP frequency from RR to the theta band in CA1 and PFC, reflecting the change in behavioral state (Supplementary Fig. 2g). and $\mathrm{RR}$ in all three regions, consistent with previous findings ${ }^{53}$ (Supplementary Fig. 2h), and suggesting a mechanistic relationship between the two rhythms. Despite this relationship, previous literature shows that the two rhythms are differentially generated and maintained in the olfactory system and hippocampus and appear to serve different functions in olfactory processing ${ }^{66-69}$. This indicates that the beta rhythm is not merely a harmonic of RR despite apparently similar modulations in amplitude during this task. 
142 prominent and consistent coherence at the beta frequency band during odor sampling, prior to

143 the decision execution at odor-port offset (Fig. 2c). This coherence was specific to the odor-

144 sampling period: it significantly increased compared to time-matched pre-odor periods (Fig. 2d,

145 sign-rank test, $p=6.7 e-6)$, and diminished shortly after the rat exited the odor port, once the

146 decision had been made (Fig. 2c). Additionally, we observed coherence in the RR band in the

147 CA1-PFC circuit, although there was no significant change between pre-odor and odor-sampling

148 periods (Fig. 2d, signed-rank test, $p=0.36$ ). During running, after the decision-making period,

149 the prominent low frequency RR coherence shifted to be slightly higher and centered on the

150 canonical theta band $(6-12 \mathrm{~Hz})$, similar to what was observed in the LFP power spectrum

151 (Supplementary Fig. 3a). Beta and RR coherence were also observed between the CA1-OB

152

153

and PFC-OB regions during odor sampling periods, with similar temporal dynamics as CA1-PFC beta coherence (Supplementary Fig. 3b-c).

To directly examine the influence of rhythmic coordination on task dynamics, we asked whether the animal's decision latency, indicated by odor port exit time, was linked to the degree of network coordination. A significant negative correlation between the decision latency time and CA1-PFC beta coherence indicated that a higher degree of beta coordination in the network is associated with faster decisions, whereas there was no significant correlation between RR coherence and decision latency time (Fig. 2e, beta: $r=-0.54, p=4.4 e-4$; RR: $r=0.03, p=$ 0.87). Similarly, CA1-OB beta coherence, but not RR coherence, was also correlated with decision latency (Supplementary Fig. 3d), but there was no significant relationship between decision latency and PFC-OB coherence in either frequency band (Supplementary Fig. 3e).

To control for the possibility that elevated beta coherence during the decision-making period was simply a reflection of passive movement preparation, as has been observed in sensorimotor cortex ${ }^{70,71}$, we compared CA1-PFC beta coherence during the last $500 \mathrm{~ms}$ of the decision-making period with the $500 \mathrm{~ms}$ just prior to reward well exit. We confirmed that beta 
coherence observed during the decision-making period was significantly higher than coherence at the reward well. (Fig. 2f, signed-rank test, $p=1.2 \mathrm{e}-7$ ). In an additional control, we also confirmed that this elevated beta coherence was specifically seen during odor-cued decision making and was not present in sessions in which only air was presented at the odor-port (see Methods). In these air-only sessions, reward was given on random trials at the reward locations, even though no odors were presented. RR coherence was unchanged between odorand air-cued sessions, but beta coherence was significantly lower during air-cued sessions

\section{(Supplementary Fig. 3f-g).}

Although we found a strong relationship between beta coherence in the CA1-PFC network and decision latency, we did not find a direct relationship between the strength of beta coherence or RR coherence to correct performance on the task. There was no significant change in the level of coherence in the CA1-PFC network in either frequency band between correct and incorrect trials (Fig. $\mathbf{2 g}$, signed-rank tests; beta: $p=0.38$; RR: $p=0.81$ ). This pattern was true of CA1-OB coherence (Supplementary Fig. 3h), as well as PFC-OB coherence (Supplementary Fig. 3i). Additionally, although beta power was higher on correct trials compared to incorrect trials in PFC, this was not true of either CA1 or OB (Fig. $\mathbf{2 h}$ (top row), signed rank test; CA1: $p=0.08$; PFC: $p=9.3 e-4$; Supplementary $3 \mathbf{j})$. RR power was unchanged between correct and incorrect trials in all three regions (Fig. $\mathbf{2 h}$ (bottom row), bootstrap test; CA1: $p=0.26$; PFC: $p=0.16$; Supplementary $3 \mathbf{j})$. Therefore, while beta coherence in the CA1-PFC network may facilitate quicker decision making, the strength of oscillatory coordination in the network may not directly or inherently enable a correct decision suggesting that oscillatory modulation of cellular activity may instead play a role.

We were curious if these beta rhythm dynamics were limited to the odor sampling location on the maze. Indeed, CA1-PFC network beta power and coherence were clearly isolated to the region near the odor port (Fig. 2i). Finally, since hippocampal sharp-wave ripples 
(SWRs) have been proposed as a mechanism for memory retrieval, decision making, and planning in some tasks ${ }^{13,72-74}$, we also investigated the occurrence of SWRs during our task. SWR events occurred frequently at the two reward locations, as expected ${ }^{75}$. However, a distinct lack of SWR events at the odor port (Figs. $\mathbf{2} \mathbf{i}$ and $\mathbf{2} \mathbf{j}$; signed-rank test, $p=1.1 \mathrm{e}-7$ ) suggests that SWR replay is unlikely to play a direct role in decision making based on recalled associative memories for this well-learned task; rather, the beta rhythm appears to be a primary coordination mechanism for this function. Together, these results suggest that the beta rhythm specifically plays a principal role in odor-cued decision making in this task, while RR may play a more passive role in organizing sensory information transfer during cue utilization. interneurons; PFC: $87 \%$ pyramidal, 13\% interneurons). Pyramidal cells were then further categorized as task-responsive or task-unresponsive based on whether they exhibited significant changes in firing rate during odor sampling (see Methods; $n=125$ task-responsive CA1 cells, $n=157$ task-responsive PFC cells). We observed an overall increase in spiking

211 during the odor sampling period in the task-responsive cell population, suggesting that the 212 majority of these cells increase their firing rate during this period (Supplementary Fig. 4a).

213 Interestingly, however, there was an overall decrease in spiking in the average peri-stimulus

214 time histograms when pooling all spikes from all cells during the odor-sampling period

215 (Supplementary Fig. 4b), suggesting a decrease in overall responsiveness in CA1 and PFC 
networks during odor sampling. Additionally, we confirmed that task responsive neurons were

217 not simply modulated by the animals' decrease in speed at the odor-port by comparing firing

218 rates during odor sampling to firing rates during reward consumption, when speed was also low.

219 We found task-responsive cells' firing rates were significantly higher at the odor port than at the

220 reward wells (Supplementary Fig. 4c).

A subset of task-responsive cells was selective for specific choices, based on the

222 identity of the odor and the associated choice. Selectivity was calculated by generating a selectivity index (SI), in which the difference between the average firing rate response to each odor on correct trials was divided by the sum of the two responses, giving a value between -1 we observed was relatively low, these numbers were consistent with other studies examining single unit responses to odor stimuli in these regions ${ }^{8,76,77}$.

Peri-stimulus time histograms and raster plots aligned to decision time for two example selective cells for CA1 and PFC are shown in Figs. $\mathbf{3 b}$ and $\mathbf{3 g}$ respectively. Notably, we found that on incorrect trials, selective cells often exhibited responses to the two odors that were opposite to the responses on correct trials. (Examples shown in Figs. $\mathbf{3 c}$ and $\mathbf{3 h}$ ). We found that this phenomenon held true across the population of selective cells, such that overall, the SIs on correct trials were anti-correlated with the SIs on incorrect trials (Figs. $\mathbf{3 d} \mathbf{d}$-e and $\mathbf{3 i} \mathbf{i} \mathbf{j}$ ). Note that since there are only two possible choices on the task, the behavioral response on an

237 incorrect trial is identical to that of a correct trial of the opposite odor. The fact that the neural responses on trials with identical behavioral responses are similar indicates that selective cells 
241 alone would result in similar responses to a given odor regardless of the upcoming behavior or

242 the ultimate trial outcome. We therefore termed these cells choice-selective cells. This pattern

243 did not persist within the population of neurons that were task-responsive but did not meet the

244 selectivity threshold (Supplementary Fig. 4d-e). Histograms showing distribution of absolute

245 selectivity indices between selective and nonselective cells are shown in Supplementary $\mathbf{4 f .}$

246 Putative interneurons were also divided into task-responsive and task-unresponsive,

247 using the same criteria as for putative pyramidal cells (Supplementary Fig. $\mathbf{4 g}$ ).

248 Supplementary Fig. 4h-i shows interneuron odor selectivity indices and response properties.

249 For CA1 interneurons, the selectivity response was similar to that of pyramidal cells. PFC

250 interneurons' SIs on correct and incorrect trials were not anti-correlated, although there were

251 very few selective PFC interneurons overall.

Neural ensemble responses during decision making are governed by beta coordination and predict the upcoming choice

We next examined how ensemble dynamics underlie the neural representation of decisions informed by odor-cued recall. We considered all cells that were task-responsive

257 (regardless of the significance of SI for individual cells) for the ensemble analyses. For the

258 population of task-responsive cells, we found that the distribution of the peak response times in

259 both CA1 and PFC tiled the entire decision time window (Fig. 4a). Using the population

260 differential index (PDI) as a measure of population response discriminability (see Methods), as

261 described previously ${ }^{5}$, we found that distinct representations for the two possible choices

262 emerged gradually in the neural population during the decision-making time period (Fig. 4b).

263 Briefly, for this analysis, all task-responsive cells were pooled across animals and days into a

264 single population. Mean firing rates were calculated in $100 \mathrm{~ms}$ bins to obtain population vectors 
(PVs) for each of the two odors, and at each time bin the correlation coefficient between the two PVs was calculated. This correlation coefficient was subtracted from 1 to obtain the PDI, such that PDI = 1 indicates that the population responses for the two odors are completely distinct, whereas PDI $=0$ indicates that the responses are identical. Chance-level PDI was computed by shuffling the odor identities across trials and creating a null distribution.

To determine when this discriminability emerged in the CA1 or PFC ensembles, we performed a bootstrap test, in which a subset of 50 neurons from each region was randomly sampled with replacement to use for the PDI calculation. The timepoint at which the population responses to the odors were considered significantly distinct from each other was defined as the first timepoint at which the real PDI surpassed the 95\% confidence interval of the null (shuffled) distribution. This procedure was repeated 500 times to generate a distribution of discrimination times for each region, aligned to odor port disengagement. These bootstrapped distributions were compared, which suggested that discriminability in the CA1 and PFC ensembles emerged simultaneously (Fig. 4c, CA1: median $=0.19$ seconds prior to odor-port disengagement, interquartile range $=0.06$; PFC: median $=0.23$ seconds prior to odor-port disengagement, interquartile range $=0.06$; bootstrap test, $p=0.31$ )

We were next interested in whether the timing of neural discrimination for CA1 and PFC ensembles on each trial (determined as the time of peak PDI discrimination value) was related to the decision latency. We determined the timing of peak PV discrimination in each region on individual trials by comparing the task-responsive PVs on each trial to the average PV on trials with the opposite odor. Trials in which fewer than 3 total task-responsive neurons in the region were active were excluded, since at least three datapoints are needed to calculate a meaningful correlation between PVs. On individual trials, the timing of peak discrimination for both CA1 and PFC ensembles had small but significant correlations with the decision latency (Fig. 4d, CA1: $n$ $=2241$ trials, $r=0.09, p=4.1 e-5 ;$ PFC: $n=2087$ trials, $r=0.06, p=0.01$; individual trial rasters 
shown in Supplementary Fig. 4j). This implies a temporal link between the emergence of the decision in the CA1 and PFC neural populations, and the moment when the animal begins to execute a behavioral response.

We next asked whether the timing of this neural ensemble discriminability between the two choices was related to beta coordination in the network on each trial. Indeed, we found that the timing of peak CA1 PV discriminability on individual trials was weakly but significantly anticorrelated with the strength of CA1-PFC beta coherence, such that high CA1-PFC coherence trials had an earlier PV divergence latency and therefore earlier neural discrimination; however, no significant correlation was seen for PFC PV divergence time. (Fig. 4e, CA1: $r=-0.12, p=$ $=0.04, p=0.04)$, further suggesting a temporal relationship between beta coherence in the CA1-PFC network and coding of the choice by neural ensembles.

To determine whether the animal's upcoming behavior could indeed be predicted by neural ensembles before the decision was executed, we trained a generalized linear model 306 (GLM) to predict the animal's choice based on ensemble activity during the decision-making period (See Methods). We performed this analysis using task-responsive putative pyramidal cells and found that reward choice could be accurately predicted 0.1 seconds prior to odor port disengagement by CA1 ensembles, and 0.3 seconds prior by PFC ensembles (Fig. 4f). Additionally, we performed the same analysis, but included both task-responsive pyramidal and

311 interneurons in the ensembles. In this case, we found an improvement in prediction for CA1

312 ensembles: reward prediction was now accurate starting earlier, at 0.2 seconds prior to decision 313 execution. Prediction by PFC ensembles remained the same. (Fig. $\mathbf{4 g}$ ). To control for the 314 possibility that inclusion of interneurons improved prediction latency for CA1 simply due to the 
larger number of cells in the training set, we performed this analysis again by resampling the pyramidal cells to match the total number of pyramidal cells plus interneurons used in Fig. $\mathbf{4 g}$.

317 In this control analysis, we found that the choice could again only be predicted at 0.1 seconds

318 prior to the decision execution, the same as what we observed with the original sample of

319 pyramidal cells. These results confirm that the animals utilize the recalled odor-place association and make a spatial choice during this odor-sampling period, which is reflected in the activity of task-responsive neural ensembles. Further, beta coordination in the CA1-PFC network is linked to the neural activity dynamics underlying this memory-guided decision process.

To investigate whether there was evidence of coordination between ensembles in CA1 were temporally linked ${ }^{78,79}$. In order do this, we computed the Z-scored cross-correlation for all of the peaks of the cross-correlations for pairs of putative pyramidal cells $(n=603$ pairs) was bimodal, with a peak at $-28.5 \mathrm{~ms}$ and another at $13.3 \mathrm{~ms}$. This points to potential bi-directional spike-time coordination between the pyramidal ensembles in the two regions. Since we also found task-responsive interneurons that contributed to prediction of upcoming choice, we hypothesized that inter-regional coordination may also be shaped by interneuron activity. To

334 procedure for all CA1-PFC pairs of task-responsive interneurons ( $\mathrm{n}=85$ pairs), as well as pairs 335 of CA1 interneurons and PFC pyramidal cells $(n=462$ pairs), and vice versa $(n=130$ pairs).

336 We found a prominent peak at 0ms for CA1-PFC interneuron pairs, as well as CA1 pyramidal 337 cell - PFC interneuron pairs. Additionally, we found a significant $9.5 \mathrm{~ms}$ lag in the CA1 338 interneuron- PFC pyramidal cell cross-correlation peak distribution. These results suggest temporal relationships between these populations, and especially that CA1 interneuron activity 
may have a role in sculpting PFC task-responsive ensembles, further suggesting coordination between the two regions during cue-processing and decision-making.

\section{CA1 and PFC cells phase lock to beta and respiratory rhythms during decision making}

We next asked if there was any relationship between oscillatory phase modulation of cells and decision accuracy. Within the population of task-responsive cells in both CA1 and PFC we found a fraction of cells that were phase locked to the beta rhythm during the decisionmaking period (see Methods; CA1: $n=36,28.8 \%$; PFC: $n=17,10.8 \%$ ). Spike-phase histograms for two example beta-phase locked cells are shown in Fig. 5a (Rayleigh Z test, CA1: $n=242$ spikes, $z=15.4, p=1.5 e-7 ;$ PFC: $n=99$ spikes, $z=4.9, p=0.007)$. We also found that many cells in the task-responsive populations were strongly phase locked to RR $(C A 1: n=$ 112, 89.6\%; PFC: $n=60,38.2 \%)$. Examples for two of these cells are shown in Supplementary Fig. 5a. For both beta and RR, there was a significant non-uniformity in beta phase-preference across cells in CA1, and nonuniformity in only beta-phase preference for PFC populations (Fig. 5b, Rayleigh $Z$ test; CA1: $z=7.88, p=3.4 e-4$; PFC: $z=4.9, p=0.007$. Supplementary Fig. 5b). The preferred phases of the two cell populations were distinct for both regions (Fig. 5b, Watson-Williams test, $p<0.001$, Supplementary Fig. 5b).

Since our results so far suggested that the beta rhythm may have more relevance to decision latency and ensemble coordination than $R R$, we next asked whether phase locking to the beta rhythm was indicative of accurate decision making. To do this, we compared Rayleigh Z scores, a measure of non-uniformity in the spike-phase distribution, on correct versus incorrect trials for significantly phase-locked cells. For each phase-locked cell, we calculated the Rayleigh Z score for the spike-phase distribution on correct trials for incorrect trials separately and compared these two paired distributions. We found that in both CA1 and PFC, cells that 
were phase-locked to the local beta rhythm exhibited a lower Rayleigh Z on incorrect trials. There was no significant effect for cross-regional modulation however (Fig. 5c, signed-rank tests, CA1-CA1: $n=28, p=0.03$; PFC-PFC: $n=8, p=0.007$; CA1-PFC: $n=18, p=0.39$; PFCCA1: $n=12, p=0.20)$. This suggests that local spiking modulation by the beta rhythm in CA1 and PFC can potentially play a role in supporting accurate utilization of odor-place associations for making decisions. CA1 cells that were phase-locked by local CA1 RR also showed a slight but significant decrease in modulation on incorrect trials, but we found no change in modulation strength for locally phase locked PFC cells, nor for cross regional RR phase modulation in either population between correct and incorrect trials (Fig. 5d, signed-rank tests, CA1-CA1: $n=103$ cells, $p=0.04 ;$ PFC-PFC: $n=35$ cells, $p=0.38 ;$ CA1-PFC: $n=85$ cells, $p=0.26 ;$ PFC-CA1: $n=$ 49 cells, $p=0.30$ ). Note that the LFP and spiking analyses found stronger evidence that beta coordination is related to task performance (Figs. 2, 4, 5c), even though a higher percentage of cells in both regions were phase locked to RR (Fig. 5e, z-test for proportions, CA1: $p=6.8 e-23$; PFC: $p=8.8 e-9)$ and this modulation was much stronger for RR compared to beta (Supplementary Fig. 5c). Additionally, in both CA1 and PFC, the proportion of cells that were phase locked to both beta and RR was no different than chance, given the percentages of cells that were modulated by either rhythm (Supplementary Fig. 5d). This suggests that the two rhythms simultaneously modulate slightly different populations of cells, supporting the interpretation that the two rhythms play separate, though cooperative, roles in memory-guided decision making.

Are cells that are modulated by beta the same population of cells that code for the upcoming decision? To test this, we compared the number of pyramidal cells that were both choice-selective and phase-locked (within and across regions) to the fraction that would be expected by chance, given the total percentages of cells that are choice-selective or phaselocked. Interestingly, we found that the number of cells that met both criteria was no different 
than chance for either CA1 or PFC (Fig. 5f, binomial tests; CA1: $p=0.097$; PFC: $p=0.17$ ). We found similar results for RR modulation (Supplementary Fig. 5e). It should be noted that since such a large majority ( $90 \%)$ of neurons in CA1 were significantly phase locked to $R R$, it is unsurprising that almost all the choice-selective cells were phase locked to this rhythm. However, this result indicates that the choice-selective cells were no more or less likely than non-selective cells to phase lock to RR. Together, these results suggest that, surprisingly, the putative pyramidal neural ensembles that code for the primary task parameter of odor-cued associative decisions may not be driven directly by the beta rhythm, with relatively low prevalence of beta phase modulation, despite the strong relationship we observed between beta oscillations, choice-selective ensemble activity and memory-guided decision latency (Figs. 2, 4).

We therefore examined the role of beta oscillations in coordinating the activity of interneurons in the CA1-PFC network. Indeed, we found high prevalence of beta phase modulation of CA1 interneurons. Of the 102 interneurons in CA1 that were active during the decision period, $44(43.1 \%)$ were phase-locked to the local CA1 beta rhythm, and $25(24.5 \%)$ were phase-locked cross-regionally to the PFC beta rhythm. Phase preference among the neurons in both groups was strongly non-uniform and unimodal (Fig. $\mathbf{5 g}$ ), indicating local- and cross-regional coordination of CA1 interneurons is governed by beta in a strongly phasedependent manner. In contrast, very few of the 37 PFC interneurons were phase-locked to beta, 408 both locally (3 units, $8.1 \%$ ) and cross-regionally (1 unit, $2.7 \%$ ). incorrect compared to correct trials (Fig. 5h, signed-rank tests, CA1-CA1: $p=8.2 e-9$; CA1-PFC:

$411 p=1.2 e-5)$, suggesting that entrainment of CA1 interneurons to the network-wide beta rhythm is

412 linked to task performance. About half of the CA1 interneurons that were choice-selective were 413 also beta phase-locked, although this number was not different than what would be expected by 
chance (Fig. 5i, binomial tests; CA1: $p=0.11$; PFC: $p=0.20$ ), similar to what we observed in the population of putative pyramidal cells. Our results suggest, therefore, that CA1 interneuron activity is strongly governed by the beta rhythm locally, as well as in a coordinated manner with

417 PFC. This also aligns with the finding of temporal relationships in cross-correlations between

418 CA1 interneurons and PFC pyramidal cells and interneurons (Fig. 4h). Beta modulation of

419 interneurons may thus constrain and coordinate network activity, and may play a role in

420 sculpting pyramidal cell ensembles in local circuits to enable processing of odor-cued

421 associations for translation to decisions.

\section{Representations of choice and space are maintained independently during delay period}

Finally, we investigated if there was any relationship between the activity of CA1 and

425 PFC ensembles during the odor-cued decision-making period and their spatial activity on the maze as the animals ran through the delay period on the central and side arms toward reward.

427 We examined units from sessions in which animals traversed a long T-maze track (see

Methods, 26 sessions from 6 rats) in order to assess spatial firing characteristics. A large on spatial parameters, $n=344$ out of 585 CA 1 cells; $n=159$ out of 288 PFC cells had fields on the track), including units that were both task-responsive (choice selective and non-selective) spatial fields shown in Supplementary Fig. 6a). 
PFC: $p=2.7 e-5)$. Additionally, for both CA1 and PFC neurons, we found that the firing rate during odor sampling was correlated with firing rate during running (Fig. 6c, CA1: $r=0.39, p=$ 3.0e-37; PFC: $r=0.68, p=1.2 e-62)$, and task-responsive cells had higher firing rates overall

(Supplementary Fig. 6b), suggesting a possible relationship between cell activity during decision making and maintenance of the decision during the spatial delay. To examine this question further, we asked whether the spatial fields in either CA1 or PFC exhibited different 444 characteristics based on whether they were responsive during the decision-making period.

445 Surprisingly however, we found no difference in field width (Fig. 6d, rank-sum tests, CA1: $p=$ 0.15; PFC: $p=0.92$ ), field peak rate (Supplementary Fig. 6c), or field sparsity

(Supplementary Fig. 6d) between cells that were task-responsive and task-unresponsive in either region.

Further, we also examined trajectory selectivity as animals ran through the central arm and on the side arms toward reward (examples of trajectory selective firing shown in Fig. $\mathbf{6 a}$, second row). Trajectory selectivity was defined by comparing spatial tuning curves of cells on right versus left trajectories and calculating a selectivity index analogous to the choice selectivity index during the decision-making period (see Methods). Interestingly, although trajectoryselective cells were significantly more likely than trajectory-nonselective cells to respond in the decision-making period (Supplementary Fig. 6e), there was no relationship between the preferred trajectory during run periods and preferred choice during the odor-cued decisionmaking period (Fig. 6e, rank sum test CA1: $p=0.26$; PFC: $p=0.20$ ). Thus, although CA1 and PFC cells that were task responsive had higher firing rates and therefore higher engagement 459 while traversing trajectories on the maze, there was no clear relationship between choice 460 selectivity and trajectory selectivity in either CA1 or PFC. 
this, the linearized T-maze was divided into 5 equally spaced quintiles and a Bayesian Classifier trained on odor period activity was used to decode the choice identity from the ensemble activity during run at each spatial quintile (see Methods; similar results were seen for spatial quartiles). We found that choice identity could be decoded at the first quintile of track, nearest to the odor

467 port, but decoding accuracy diminished to chance level thereafter (Fig. $\mathbf{6 g}$ ). In contrast, the 468 animal's upcoming behavioral choice could be accurately decoded at all periods along the 469 trajectory if instead the spatially active ensembles in each respective spatial quintile was used as the training set (Fig. 6h). This suggests that the choice selectivity that emerges in ensembles during odor sampling (Fig. 4) persists transiently for a short period past the decision point, but

472 that separate ensembles maintain choice-related information as animals traverse the spatial

473 trajectory, possibly corresponding to theta oscillations during run (Supplementary $\mathbf{2 g}$ ). This is

474 further supported by the fact that, at least for CA1, choice-selective ensemble firing rate is

475 highest within $20 \mathrm{~cm}$ of the odor port, and decreases thereafter (Supplementary Fig. 6f). These 476 results together suggest that the choice-selective ensembles reflecting decision making during 477 the odor-cue period transiently encode the choice during the initial track period, but the decision 478 is subsequently maintained by a distinct ensemble during the working-memory period associated with the spatial delay on the central arm.

\section{DISCUSSION} hippocampal-prefrontal ensembles by beta rhythms for odor-cued associations and decision making. We found that during an odor-place associative memory task, beta rhythms govern physiological coordination between the olfactory bulb, hippocampus, and PFC. During the odor sampling and decision-making period, task-responsive single unit and ensemble activity in the 
Further, CA1-PFC ensemble dynamics and beta coherence during this period are linked to latency of decisions. Despite this link, we did not find evidence for direct beta phase modulation of choice-encoding putative pyramidal neurons. Instead, we found that interneurons in CA1 were strongly locally and cross-regionally phase modulated by beta. This suggests that coordination by beta rhythms may sculpt network activity through interneuron modulation, enabling emergence of task-related ensemble dynamics in local circuits to support decision

494 making. Our results thus suggest that beta oscillations play a key modulatory role in enabling

495 temporal evolution of ensemble dynamics in the CA1-PFC network for odor-cued decision 496 making, delineating a novel mechanism through which rhythmic coordination supports cognition. behavior involves widespread networks encompassing sensory regions, medial temporal memory regions, and prefrontal executive function regions. Prominent odor-driven oscillations have been described in these areas ${ }^{5,47-53,80}$, which can potentially coordinate these long-range networks to enable utilization of familiar olfactory cues to guide behavior. Here, the use of an odor-cued T-maze task allowed us to examine which rhythms enable coordination of olfactoryhippocampal-prefrontal networks, and whether these rhythms play a role in patterning ensemble activity in hippocampal-prefrontal network to enable memory-guided decision making. temporal window corresponding to odor-cued recall and priming of the subsequent decision to turn toward the reward location. We found increases in power and coherence of both beta $(\sim 15-$ $30 \mathrm{~Hz})$ and $\mathrm{RR}(\sim 7-8 \mathrm{~Hz})$ across the olfactory-hippocampal-prefrontal network during this odor

511 sampling and decision-making period. However, only the strength of beta coherence, and not

512 RR coherence, between the hippocampus and PFC was correlated with shorter decision 
513 latency. Further, while CA1-PFC RR coherence remained unchanged between odor-cued trials

514 and trials where only air was presented, high beta coherence was specific to odor-cued trials

515 corresponding to a decision-making task and was lower on air-cued trials corresponding to

516 random choices. Based on these findings, we speculate that encountering a familiar odor

517 stimulus and efficiently utilizing this associative memory for a decision, rather than respiration or

518 movement preparation ${ }^{81}$, elicits the engagement of the hippocampal-prefrontal network by the

519 beta rhythm.

Interestingly, although hippocampal SWRs have been linked to internal recall and

planning ${ }^{72,73,82}$, there was very low prevalence of hippocampal SWRs during odor sampling,

527 Therefore, the lack of SWRs during the decision-making period could reflect that these memories had been previously consolidated into neocortex during learning, resulting in a shift from largely hippocampal-dependent processing ${ }^{5}$ to processing that is more reliant on hippocampal-cortical dialogue via the beta rhythm. tasks $47,66,83$, and may play a complementary role with beta oscillations. It has been hypothesized that local olfactory networks may be governed by gamma rhythms for odor processing early in

534 stimulus sampling, before shifting to a beta-dominant network state as downstream regions are engaged ${ }^{47,84}$. Our results corroborate this hypothesis by reinforcing the role of the beta rhythm in 
538

539

540

541

542

543

544

545

546

547

548

549

550

551

552

553

554

555

556

557

558

559

560

561

562

general role in cue-driven mnemonic decision making by coordinating sensory and cognitive areas, which can be tested in future studies.

Our analysis of single units in the hippocampus and prefrontal cortex revealed a group of cells that were selectively responsive in the odor task period, similar to previous reports in the hippocampus ${ }^{5,85,86}$. However, we found that these selective cells often exhibited "opposite" selectivity on incorrect trials compared to correct trials, indicating that these cells do not respond solely to the perceptual qualities of the odor, in which case the response would be similar on all presentations of a particular odor regardless of the trial outcome. Instead, this response suggests coding of the behavioral choice associated with the odor cue, resembling findings from studies of combined cue modalities and cue-context associations ${ }^{1,6-8,10,86-88}$. Thus, the CA1-PFC neural response during odor sampling corresponds to the decision in response to the odor-place association. Future studies that combine recording from other olfactory regions, such as piriform cortex, together with hippocampus and PFC can potentially unveil how the cue representation evolves from odor perception to the recall-based decision.

Task-responsive neural populations exhibited differential activity patterns in response to the two odor-associations during decision making, and the animal's upcoming choice could be predicted from this ensemble activity. Correlation between the timing of ensemble odor discrimination and choice execution indicates that the emergence of the neuronal representation coincides with and potentially primes the cognitive decision. This emergence of neural ensembles that reflect associative memories governing decision making may also be indicative of a physiological mechanism for dynamic reactivation of memory engrams upon reencountering the familiar odor stimuli ${ }^{89,90}$. We also found evidence that beta rhythms may govern the temporal evolution of CA1-PFC neural ensemble dynamics leading to memoryguided decision making, as demonstrated by the small but significant correlations between the strength of beta coherence, the timing of ensemble discrimination, and the behavioral decision 
563

564

565

566 567 task $^{5}$.

568

569

570

571

572

573

574

575

576

577

578

latency. It remains unclear, however, how these neural dynamics and their relationship to the beta rhythm evolve as animals learn new associations. One possibility is that they resemble the dynamics observed in the hippocampal-entorhinal circuit, in which coordination of the network at the beta rhythm and choice-selective neural activity emerge gradually as animals learn the

Similarly, CA1-OB beta coherence is strong during odor memory tasks and is known to increase over the course of learning ${ }^{58}$. Although CA1-OB beta coherence was high during odor sampling in our task, we observed no significant relationship between CA1-OB coherence and task performance. Since previous reports suggest that this coherence is linked to the learning process, it is possible that a more direct relationship between coherence strength and performance would be observed only during learning, but not in this case where the odor association and task are familiar. Choice-coding ensemble dynamics may evolve in parallel with the increase in $\mathrm{CA} 1-\mathrm{OB}$ beta coherence over learning, similar to what has been reported in the hippocampal-entorhinal circuit 5 . This possibility can be investigated by examining network dynamics and ensemble activity across multiple olfactory and cognitive areas during novel odorplace learning or reversal learning.

It is also noteworthy that we observed different mechanisms underlying odor-cued decision making versus subsequent maintenance of the representation of the choice during a spatial delay period. Odor-driven beta coherence leads to emergence of ensemble selectivity during the decision-making period, which is predictive of the animal's upcoming choice, but these ensembles maintain their choice-selectivity only transiently after the decision execution as animals embark on the spatial trajectory toward reward. This selective coding is not maintained by the same ensembles during running, but instead other spatially modulated cells in CA1 and PFC maintain choice coding on the track, including the spatial delay period (common central arm on the T-maze) activity. Thus, beta-driven ensembles mediate memory-guided decision 
588

589

590

591

592

593

594

595

596

597

598

599

600

601

602

603

604

605

606

607

608

609

610

611

612

making in the hippocampal-prefrontal network, but choice selective activity is then subsequently maintained by theta-driven spatially modulated activity, similar to reports in spatial working memory tasks ${ }^{77}$.

The temporal evolution of ensemble activity underlying odor-cued decision making, and its relationship to beta oscillations, is reminiscent of temporal coding of odor stimuli mediated by oscillations in the olfactory system ${ }^{50,52}$. Previous findings indicate that spike timing modulation according to the phase of prominent network rhythms can organize task-encoding neural ensembles to support memory guided behavior and decision-making ${ }^{42,86,91-94}$. Interestingly however, we found that although the strength of local beta and RR phase-modulation was linked to correct decisions, putative pyramidal neurons that encoded the choice were no more likely to be phase-locked than chance level. Notably, phase locking was stronger and more prevalent for RR compared to the beta rhythm, even though behavioral decision latency and choice-coding in the underlying ensembles was more linked to beta coherence in the network. Further, we found evidence that slightly different populations of cells are modulated by the two ongoing rhythms during decision making, implying that beta and RR represent two simultaneous modes of rhythmic coordination in the network ${ }^{49}$. This joint modulation of the underlying cell populations, along with our finding of strong cross-frequency coupling between the two rhythms, leads us to the interpretation that beta and RR coordinate the network cooperatively during odor-cued decision making, rather than acting as independent but parallel processes. Previous reports of $\mathrm{RR}$ in olfactory processing ${ }^{51,52,55}$, along with evidence that multiple rhythms can simultaneously modulate ongoing processes ${ }^{49,95,96}$, suggests that $\mathrm{RR}$ is the dominant modulator coordinating the sensory element of the task, while beta coordination is key for employing the sensory cued association to make a decision. This interpretation is further supported by our results from uncued air sessions, which did not involve memory-guided behavior, in which we found a reduction in beta coherence in the network but no change in RR coherence. This suggests that 
613 the sensory component of this process is maintained as the animal continues sniffing even in

614 the absence of an explicit cue, whereas beta coordination is only engaged when a familiar cue

615 is utilized for a decision.

616 In contrast to pyramidal cells, we found that a large population of CA1 interneurons were

617 strongly modulated by the beta rhythm locally and cross-regionally and the strength of this

618 modulation was linked to task performance. This suggests that the beta rhythm can function by

619 modulating CA1 interneuron activity, which sculpts ensemble dynamics in the local circuits

620 governing decision making. In this manner, beta oscillations may play a role in establishing

621 communication and organization of activity in sensory and cognitive networks enabling

622 decisions based on cued associations. Thus, oscillatory phase-modulation may be indicative of

623 a general network state that enables coordination. 


\section{METHODS}

625

626

627

628

629

630

631

632

All experimental procedures were approved by the Brandeis University Institutional Animal Care and Usage Committee (IACUC) and conformed to US National Institutes of Health guidelines. Eight male Long-Evans rats (3-6 months, 450-650 g) were used for experiments. Animals were housed individually in a dedicated climate-controlled animal facility on a 12-hour light/dark cycle. All experiments were carried out during light cycle. Upon arrival, animals were provided ad libitum access to food and water and handled regularly to habituate them to human contact.

\section{Behavior Apparatus}

An olfactometer (MedAssociates Inc.) was used for dispensing odors. The olfactometer continuously dispensed clean air to the odor port until receiving a signal to open a solenoid valve which caused air to flow through liquid odorants, resulting in odorized air dispensed to the odor port. A vacuum tube attached to the odor port was used to continuously collect any residual odorized air between trials. Infrared beams were used at the odor port and reward wells to determine the precise timing of entry and exit from these areas.

\section{Odor-Place Association Task Training}

Once rats reached a minimum threshold weight of $450 \mathrm{~g}$, they were food restricted to no less than $85 \%$ of their free-feeding baseline weight. For initial behavioral training, rats were familiarized with the behavior room, the sleep box, running on a raised track to receive evaporated milk reward, and sniffing odors presented in the odor port. Following this habituation, rats were trained to hold their nose in the odor port for a minimum of $500 \mathrm{~ms}$, with 
647

648

649

650

651

652

653

654

655

656

657

658

659

660

661

662

663

664

665

666

667

668

669

670

671

an auditory tone indicating when this $500-\mathrm{ms}$ time threshold was reached. However, the rats could continue to sniff the odor for any longer duration of time, and odor would be continuously dispensed until they disengaged from the odor port. Throughout all training and experiments, if the rat exited the odor port before this threshold was reached, no reward was dispensed regardless of the rat's choice and the rat was required to re-initiate the trial. These prematurely terminated trials were excluded from all analyses. For each trial, the odor sampling period was defined as the time from the onset when rat's nose broke the infrared beam to offset when the beam break was terminated, and odor stimulus was stopped.

Animals were subsequently trained on the olfactory association. On each trial, one of two possible odors was dispensed at the odor port in a pseudo-random order. Odor 1 (Heptanol - pine/citrus scent) indicated that the rat should go to Reward 1 to receive reward. Odor 2 (ethyl butyrate - strawberry scent) indicated that the rat should choose Reward 2 (Fig. 1a). If the rat ultimately made the correct choice, evaporated milk reward would be dispensed at the chosen reward port upon triggering the infrared beam, whereas upon an incorrect choice no reward was dispensed.

Associative memory training was shaped in steps. The rats first learned the association between the odors and "right" vs "left" reward locations with reward wells close to the odor port. On subsequent training days, the reward wells were moved further and further away from the odor port, until rats could comfortably perform the task on the full T-maze $(81 \mathrm{~cm}$ long center stem, and $43 \mathrm{~cm}$ long reward arms). Two rats were not trained to run on the full T-maze, and instead were just required to perform the association with the reward wells within easy reach on either side of the odor port. For one animal, the maze had a shortened stem $(40 \mathrm{~cm}$ instead of $81 \mathrm{~cm})$, but the reward wells were still at the ends of the T arms. Task performance was calculated as the proportion of correct trials. Rats were trained until they could perform the task with at least $80 \%$ accuracy for 3 consecutive days. 
Once training was complete, animals were once again provided with ad libitum access to

673 food until they reached at least $600 \mathrm{~g}$, before undergoing surgery. After surgery but before

674 recording, rats were briefly re-trained on the association until they could again perform the task

675 with at least $80 \%$ accuracy. On recording days, animals were allowed to continue performing

676 the task until they reached satiation, about 100-150 trials per day. Task epochs were interleaved

677 by sleep epochs, in which rats spent about 20 minutes in an opaque sleep box, with a sleep

678 epoch as the first and last epoch of each day.

\section{Un-Cued Air Sessions}

Four of the animals were tested on one session each in which only clean air was chosen reward port on each trial; although, since the trials were un-cued, the animals would often randomly choose the "incorrect" side and no reward was dispensed (Supplementary

Figure 3f). All other aspects of the task were the same as the odor-cued task.

\section{Surgical Procedures}


anterior to the cribriform suture. The thermocouple wire was secured using dental acrylic and

697 soldered to the same printed circuit board as the tetrodes.

\section{Tetrode Recordings}

For 1-2 weeks following surgery, tetrodes were gradually lowered to the desired depths.

701 Hippocampal tetrodes were targeted to the pyramidal layer of CA1 using characteristic EEG

702 patterns (sharp wave polarity, theta modulation) and neural firing patterns as previously

703 described ${ }^{78,97}$. The final placement of tetrodes was confirmed in histological preparations using

704 Nissl staining post-mortem. One tetrode in corpus callosum served as hippocampal reference,

705 and another tetrode in overlying cortical regions with no spiking signal served as PFC reference.

706 A ground screw (GND) installed in the skull overlying cerebellum also served as a reference,

707 and LFP was recorded relative to this GND. All spiking activity was recorded relative to the local

708 reference tetrode. Only LFP activity was recorded from olfactory bulb. Electrodes were not

709 moved at least 4 hours before and during the recording day to reduce drift, and were micro-

710 adjusted at the end of each recording day to sample new cell populations.

711 Data were collected using a SpikeGadgets data acquisition system and software

712 (SpikeGadgets LLC). Spike data were sampled at $30 \mathrm{kHz}$ and bandpass filtered between 600

$713 \mathrm{~Hz}$ and $6 \mathrm{kHz}$. LFP signals were sampled at $1.5 \mathrm{kHz}$ and bandpass filtered between $0.5 \mathrm{~Hz}$ and

$714400 \mathrm{~Hz}$. The animal's position and running speed were recorded with an overhead color CCD

715 camera (30 fps) and tracked by color LEDs affixed to the headstage. 
All data analysis was performed in MATLAB using custom code unless otherwise noted.

719

720

721

722

723

724

725

726

727

728

729

730

731

732

733

734

735

736

737

738

739

740

\section{Local Field Potential}

Trial-averaged spectrograms and coherograms were calculated using multi-taper spectral methods included in the Chronux package for MATLAB (www.chronux.org). To get the beta filtered local field potential (LFP) signal, raw LFP (with respect to GND) was band pass filtered at 15-30 Hz using a zero-phase IIR filter. Amplitude, phase, and envelope magnitude of the signals were obtained using a Hilbert transform. Beta power and coherence were z-scored to the epoch mean. Similarly, the respiratory rhythm signal was obtained by band pass filtering the raw LFP at $7-8 \mathrm{~Hz}$. Due to the overlap between the RR and the theta rhythm $(6-12 \mathrm{~Hz})$, to get an accurate measure of RR power and coherence without leakage from the theta rhythm, this signal was z-scored to time-matched periods during immobility while the animal was consuming reward.

\section{Bootstrap Tests}

Bootstrap tests for Supplementary Fig. $\mathbf{3 g}$ were performed when coherence on odorcued and air-cued trials were compared, to account for the much larger percentage of odorcued trials. Data points on odor trials were randomly downsampled with replacement to match the number of air trials, and the mean was calculated for this new set of datapoints. This downsampling was done 1000 times, to create a new, bootstrapped distribution of means. The 
observed mean value for the air trial distribution was compared to the bootstrapped distribution. P-values were calculated by counting the number of values the bootstrapped distribution that were greater than or equal to the observed mean value, and dividing by the total number of resamples (1000). P-values less than 0.05 were considered statistically significant, and thus rejected the null hypothesis that the two observed distributions had equal means. ensembles were resampled to create new ensembles of 50 cells each. The timing of PV divergence for each resampled ensemble was calculated as described below (see Population Differential Index methods). This procedure was repeated for 500 iterations to generate the distributions shown in Fig. 4c.

\section{Sharp-Wave Ripple Detection}

Hippocampal sharp-wave ripples were detected as previously described ${ }^{31,78,98}$. Briefly,

754 the locally referenced LFP signal from CA1 tetrodes was filtered in the ripple band (150-250

$755 \mathrm{~Hz}$ ), and the envelope of the ripple-filtered LFPs was determined using a Hilbert transform.

756 SWR events were detected as contiguous periods when the envelope stayed above 3 SD of the 757 mean on at least one tetrode for at least $15 \mathrm{~ms}$.

\section{Cross-frequency Coupling}

Phase-amplitude coupling between RR and beta was computed for Supplementary Fig.

$761 \mathbf{2 g}$ as previously described ${ }^{99}$. In brief, the phases of RR were divided into 20 degree bins, and

762 the mean amplitude of the beta rhythm at each phase was calculated. The mean amplitudes 
763

764

765

766

767

768

769

770

771

772

773

774

775

776

777

778

779

780

781

were then normalized by dividing each bin by the sum of amplitudes across all bins. The strength of phase-amplitude coupling was determined by comparing the amplitude distribution to a uniform distribution by calculating the modulation index (MI), which is based on the KullbackLeibler $(\mathrm{KL})$ distance but normalized so that values fall between 0 and 1 , where a value of 0 indicates a uniform distribution of beta amplitudes across RR phases. Significance was determined by comparing the calculated MI to a null distribution generated by shuffling the trial number assignments of the RR phase series; this maintains the structure of the underlying rhythm but randomly aligns the beta amplitudes on each trial to each trial sequence RR phases.

\section{Single Unit Analysis}

Spike sorting was done semi-automatically using MountainSort ${ }^{100,101}$, with manual curation. Only well-isolated units were used for analysis. Putative interneurons and pyramidal cells were classified based on average firing rate and spike width, as described previously ${ }^{31,98}$. Units were classified as interneurons if they had an average firing rate exceeding $7 \mathrm{~Hz}$ and an average spike width under $0.3 \mathrm{~ms}$. All other units were considered putative pyramidal cells. Units were excluded from analysis if they had fewer than 100 spikes across all sessions. A small number of pyramidal cells only had spikes during sleep epochs, and these were excluded from analysis ${ }^{78,102}$ since all analysis done here focused exclusively on the task periods.

\section{Task Responsiveness}

Task responsiveness was calculated as a change in firing rate following odor onset, compared to a pre-stimulus period of the same length of time as the odor sampling period on each trial leading up to odor port engagement. Sampling events were only considered if the 
animal held its nose in the odor port for longer than 0.50 seconds and proceeded to a reward

787 port. Statistical significance was determined from the Wilcoxon signed rank test of those trial-

788 matched rate differences for each odor identity separately. If a cell showed a significant change

789 up or down from baseline for at least one of the odors, it was considered task responsive. Of

790 note, there was only a single unit analyzed whose firing rate changed in opposite directions from

791 baseline for the two odors, the remainder showed the same direction of change in firing rate for

792 the two odors.

\section{Choice Selectivity}

Firing rates during odor sampling were calculated as the number of spikes as a function of

796 time from odor-port engagement to odor-port disengagement. Choice selectivity was calculated

797 using the following equation:

$$
S I=\frac{\lambda_{1}-\lambda_{2}}{\lambda_{1}+\lambda_{2}}
$$

Where $\lambda_{1}$ is the firing rate vector for Odor 1 trials, and $\lambda_{2}$ is firing rate vector for Odor 2 trials.

$800 S I=1$ indicates that the cell only responded on Odor 1 trials, whereas $S I=-1$ indicates that the

801 cell only responded on Odor 2 trials. To determine significance, a null distribution was

802 generated in which the odor identities were shuffled across trials. Cells were considered choice-

803 selective if the SI fell outside of $1.5 \mathrm{~s}$.d. from the mean of the null distribution. 
The population differential index (PDI) was calculated as described in Igarashi et al.,

807 2014. For this analysis, all task-responsive cells were pooled across animals and days into a

808 single population. Mean firing rates were calculated in $100 \mathrm{~ms}$ bins from 0 to 1 second aligned

809 to odor onset to obtain population vectors for each of the two odors. At each time bin, the

810 correlation coefficient (CC) was calculated between the Odor 1 response vector and Odor 2

811 response vector. This CC was subtracted from 1 to obtain the PDI:

$$
1-C C=P D I
$$

813 such that $\mathrm{PDI}=1$ indicates that the population responses for the two odors are completely

814 distinct, whereas PDI = 0 indicates that the responses are identical. Chance-level PDI was

815 computed by shuffling the odor identities across trials and creating a null distribution. The real

816 PDI was then compared to the null distribution across the odor sampling time window. The

817 timepoint at which the population responses considered significantly distinct from each other

818 was defined as the first timepoint at which the real PDI surpassed the $95 \%$ confidence interval 819 of the null distribution.

\section{Individual Trial PDI}

A similar PDI analysis was employed to examine population dynamics on a trial-by-trial

823 basis, in which the PDI was computed for single trials individually. Cells were not pooled across

824 animals, and only sessions with at least 3 task-responsive cells were considered for analysis.

825 The PDI was computed by calculating the CC between the trial PV and the average PV of all 826 trials of the opposite odor in that session. 
A generalized liner model (GLM) with a log link function was constructed to predict reward choice based on neural activity during the odor sampling period. Activity from all task-

831 responsive neurons that were active on at least 10 trials was included. Neural population activity

832 from different length time bins from 0.1 seconds to 1 second aligned to odor onset was used for

833 prediction, i.e. $0-0.1 \mathrm{~s}, 0-0.2 \mathrm{~s} \ldots$ 0-1.0s. Five-fold cross validation was used to test prediction.

834 For each fold, the session's trials were randomly partitioned into five equally sized sets. Four of

835 the five sets were used to train the GLM model and the remaining set was used to test. The

836 prediction accuracy was calculated by dividing the number of correctly predicted trials by the

837 total number of trials used for testing for each fold. shuffling the trial outcomes to obtain a null distribution. At each time bin used for the prediction, a rank-sum test was performed on the prediction accuracy using the real data compared to the

841 shuffled data.

\section{Phase Locking}

847 the spike phases of each cell during the odor-sampling periods and performing a

848 Rayleigh Z test for circular non-uniformity. For each cell, the mean direction of the von 


\section{Occupancy Maps}

For all spatial field analyses, only the 5 animals that were trained on the full T-maze were used; the three animals trained on the truncated maze were excluded due to insufficient spatial data. Two-dimensional occupancy maps were generated by calculating occupancy in 2$\mathrm{cm}$ square spatial bins from epochs in which the animals running speed exceeded $3 \mathrm{~cm} / \mathrm{sec}$ and

857 convolving with a $2 d$ Gaussian ( $\sigma=2$ pixels).

\section{Place Field Determination}

2-D occupancy-normalized firing rate maps were generated by dividing the spikes at each 2-D pixel by the unsmoothed occupancy at that pixel, and then and smoothing with a Gaussian kernel of $(\sigma=2$ pixels, or $4 \mathrm{~cm})$.

Linearized trajectory occupancy maps were calculated as previously described ${ }^{31,97,98}$. Briefly,

864 the rats $2 d$ spatial coordinates were first segmented into run epochs based on contiguous bouts of time in which the animals running speed exceeded $3 \mathrm{~cm} / \mathrm{sec}$. Then, the position of the animal during each 'run epoch' was categorized by its origin and destination, and each position was assigned to one of $100(1.23 \mathrm{~cm})$ bins from the origin to the destination of that route. Then the total number of spikes at each bin was divided by that bin's occupancy, and the map was convolved by a Gaussian kernel with a standard deviation of 2 pixels $(2.5 \mathrm{~cm})$.

Place field peak was calculated as the peak rate bin on the smoothed, linearized rate map, and place fields were only considered for trajectories in which the cell had a peak firing rate of at

872 least $1 \mathrm{~Hz}$. Place field width was calculated using a flood fill-algorithm in which the edges were 873 defined as the closest bins to the peak bin in which the rate fell below $25 \%$ of the peak rate. 874 Place field sparsity and information scores were calculated as previously described ${ }^{103}$. A cell 
875 was determined to have a place field if its peak firing rate along that trajectory was $\geq 2$ S.D.

876 above the mean of a bootstrapped distribution generated by circularly shifting the spikes in time

877 for each individual run, and if the field covered less than $75 \%$ of the linearized trajectory. When

878 analyzing the place field characteristics of choice-selective cells (Fig. 6, Supplementary Fig.

879 6), the field of each outbound journey was analyzed separately so as to prevent 'choosing' 880 certain fields over others.

\section{Trajectory Selectivity}

Trajectory selective cells were identified using a previously validated method ${ }^{31}$. Briefly,

884 the linearized spatial tuning curve was calculated separately for each outbound trajectory and

885 the correlation between those trajectories was computed. A cell was identified as trajectory

886 selective if this correlation value exceeded the $95^{\text {th }}$ percentile of a distribution wherein each

887 outbound trajectory identity was shuffled and the correlation recalculated.

To measure trajectory selectivity as it related to decision period selectivity, we used an analogous method to the odor selectivity index. Briefly we calculated the mean firing rate along each run beginning one half second following odor port exit and once the animals speed exceeded $3 \mathrm{~cm} / \mathrm{sec}$ to the end of that run (when the animal either reached the goal or its velocity fell below $3 \mathrm{~cm} / \mathrm{sec}$ for more than $1 / 2$ second). The difference in these mean firing rates

894 index. 

occurring in the odor sampling period. Then, the likelihood of each choice $(x)$ was reconstructed

900 from their posterior probabilities given the spikes occurring at each segment along the maze on

901 each run $\left(p(x \mid\right.$ spikes $)=p($ spikes $\mid x){ }^{*} p(x) / p($ spikes $\left.)\right)$. Run activity was defined as contiguous

902 bouts following the odor sampling period when the animal was traveling above $3 \mathrm{~cm} / \mathrm{sec}$.

903 Additionally, to prevent overlap between odor-period spiking and run spiking, the run periods

904 were defined beginning 0.5 seconds following odor port disengagement time. We assumed that

905 the $\mathrm{N}$ active cells fired independently and followed a Poisson process, giving the following

906 equation.

$$
P(X \mid \text { spikes })=C *\left(\prod_{i=1}^{N} f_{i}(X)^{\text {spikes }_{i}}\right) * e^{-\tau} \sum_{i=1}^{N} f(X)
$$

Where $\mathrm{C}$ is a normalization constant across the two choice identities. The p-value was calculated from the Gaussian statistics of a Monte Carlo random shuffle (200 shuffles) of choice

910 identity during the odor sampling period. When choice identity decoding was calculated from the

911 likelihoods of activity from each run, the decoding was performed in the same 5-fold leave-one-

912 out fashion as was used in the GLM analyses, and the $p$ value was calculated from the same

913 Monte Carlo random shuffle of the training set route identities as the odor period based 914 decoding.

\section{Data Availability}

918 The behavioral and electrophysiological data supporting the findings in this study are archived 919 on servers at Brandeis University and are available upon request to the corresponding author. 


\section{Code Availability}

922 All data processing, analyses and statistics in this study were conducted using open-source

923 package Mountainsort (https://github.com/flatironinstitute/mountainsort) and custom code in

924 MATLAB (R2018), unless otherwise noted. All custom code is available upon request to the

925 corresponding author.

926 


\section{ACKNOWLEDGMENTS}

928 This work was supported by NIH Grant R01MH120228, a Whitehall Foundation award, and a

929 Smith Foundation Odyssey award to S.P.J. We thank the late Howard Eichenbaum for

930 assistance with the behavioral task.

932 AUTHOR CONTRIBUTIONS

933 S.P.J. conceived and designed the study, C.A.S. performed experiments and analyzed the data,

934 J.B. analyzed the data, E.K. contributed to experiments, S.P.J. and C.A.S. wrote the manuscript 935 with input from all authors.

\section{COMPETING INTERESTS STATEMENT}

938 The authors declare no competing interests. 


\section{REFERENCES}

9401 Fitzgerald, J. K., Freedman, D. J. \& Assad, J. A. Generalized associative representations in parietal $941 \quad$ cortex. Nat Neurosci 14, 1075-1079, doi:10.1038/nn.2878 (2011).

9422 Wirth, S. et al. Single neurons in the monkey hippocampus and learning of new associations.

$943 \quad$ Science 300, 1578-1581 (2003).

9443 Wirth, S. et al. Trial outcome and associative learning signals in the monkey hippocampus.

$945 \quad$ Neuron. 61, 930-940 (2009).

9464 Yanike, M., Wirth, S. \& Suzuki, W. A. Representation of well-learned information in the monkey 947 hippocampus. Neuron. 42, 477-487 (2004).

9485 Igarashi, K. M., Lu, L., Colgin, L. L., Moser, M. B. \& Moser, E. I. Coordination of entorhinalhippocampal ensemble activity during associative learning. Nature 510, 143-147,

950 doi:10.1038/nature13162 (2014).

6 Allen, T. A., Salz, D. M., Mckenzie, S. \& Fortin, N. J. Nonspatial Sequence Coding in CA1 Neurons. J Neurosci 36, 1547-1563 (2016).

McKenzie, S. et al. Hippocampal representation of related and opposing memories develop within distinct, hierarchically organized neural schemas. Neuron 83, 202-215 (2014).

Otto, T. \& Eichenbaum, H. Neuronal activity in the hippocampus during delayed non-match to sample performance in rats: evidence for hippocampal processing in recognition memory. Hippocampus. 2, 323-334 (1992).

Moita, M. A., Rosis, S., Zhou, Y., LeDoux, J. E. \& Blair, H. T. Hippocampal place cells acquire location-specific responses to the conditioned stimulus during auditory fear conditioning. Neuron 37, 485-497 (2003). correct decisions during the initial learning of an alternation task. Neuron 77, 1163-1173, doi:10.1016/j.neuron.2013.01.027 (2013). 
97215 Floresco, S. B., Seamans, J. K. \& Phillips, A. G. Selective roles for hippocampal, prefrontal cortical,

973

974

975

976

977

978

17

979

980 and ventral striatal circuits in radial-arm maze tasks with or without a delay. J Neurosci 17, 18801890 (1997).

16 Battaglia, F. P., Benchenane, K., Sirota, A., Pennartz, C. M. \& Wiener, S. I. The hippocampus: hub of brain network communication for memory. Trends Cogn Sci 15, 310-318, doi:10.1016/j.tics.2011.05.008 (2011).

20 Wiener, S. I., Paul, C. A. \& Eichenbaum, H. Spatial and behavioral correlates of hippocampal neuronal activity. Journal of Neuroscience 9, 2737-2763 (1989).

21 Gothard, K. M., Skaggs, W. E., Moore, K. M. \& McNaughton, B. L. Binding of hippocampal CA1 neural activity to multiple reference frames in a landmark-based navigation task. J Neurosci 16, 823-835 (1996).

22 Hyman, J. M., Ma, L., Balaguer-Ballester, E., Durstewitz, D. \& Seamans, J. K. Contextual encoding by ensembles of medial prefrontal cortex neurons. Proc Natl Acad Sci U S A 109, 5086-5091 (2012).

23 Wiltgen, B. J., Brown, R. A., Talton, L. E. \& Silva, A. J. New circuits for old memories: the role of the neocortex in consolidation. Neuron 44, 101-108, doi:10.1016/j.neuron.2004.09.015 (2004).

24 Hasegawa, I. Neural mechanisms of memory retrieval: role of the prefrontal cortex. Rev Neurosci 11, 113-125 (2000).

25 Siegle, J. H. \& Wilson, M. A. Enhancement of encoding and retrieval functions through theta phase-specific manipulation of hippocampus. Elife 3, e03061, doi:10.7554/eLife.03061 (2014).

Fortin, N. J., Wright, S. P. \& Eichenbaum, H. Recollection-like memory retrieval in rats is dependent on the hippocampus. Nature 431, 188-191 (2004).

Cenquizca, L. A. \& Swanson, L. W. Spatial organization of direct hippocampal field CA1 axonal projections to the rest of the cerebral cortex. Brain Res Rev. 56, 1-26 (2007).

28 Ito, H. T., Zhang, S. J., Witter, M. P., Moser, E. I. \& Moser, M. B. A prefrontal-thalamohippocampal circuit for goal-directed spatial navigation. Nature 522, 50-55, doi:10.1038/nature14396 (2015).

29 Delatour, B. \& Witter, M. P. Projections from the parahippocampal region to the prefrontal cortex in the rat: evidence of multiple pathways. Eur J Neurosci 15, 1400--1407 (2002). 
100730

1008

1009

1010

1011

1012

1013

1014

1015

1016

1017

1018

1019

1020

1021

1022

1023

1024

1025

1026

1027

1028

1029

1030

1031

1032

1033

1034

1035

1036

1037

1038

1039

1040

1041

Shin, J. D. \& Jadhav, S. P. Multiple modes of hippocampal-prefrontal interactions in memoryguided behavior. Curr. Opin. Neurobiol. 40, 161-169, doi:10.1016/j.conb.2016.07.015 (2016).

31 Shin, J. D., Tang, W. \& Jadhav, S. P. Dynamics of Awake Hippocampal-Prefrontal Replay for Spatial Learning and Memory-Guided Decision Making. Neuron 104, 1110-1125 (2019).

32 Yu, J. Y. \& Frank, L. M. Hippocampal-cortical interaction in decision making. Neurobiol Learn Mem 117, 34-41, doi:10.1016/j.nlm.2014.02.002 (2015).

33 Zielinski, M. C., Tang, W. \& Jadhav, S. P. The role of replay and theta sequences in mediating hippocampal-prefrontal interactions for memory and cognition. Hippocampus, doi:10.1002/hipo.22821 (2017).

34 Place, R., Farovik, A., Brockmann, M. \& Eichenbaum, H. Bidirectional prefrontal-hippocampal interactions support context-guided memory. Nat Neurosci, doi:10.1038/nn.4327 (2016).

35 Maharjan, D. M., Dai, Y. Y., Glantz, E. H. \& Jadhav, S. P. Disruption of dorsal hippocampal prefrontal interactions using chemogenetic inactivation impairs spatial learning. Neurobiol Learn Mem 155, 351-360, doi:10.1016/j.nlm.2018.08.023 (2018).

36 Colgin, L. L. Oscillations and hippocampal-prefrontal synchrony. Curr Opin Neurobiol 21, 467474, doi:10.1016/j.conb.2011.04.006 (2011).

37 Gordon, J. A. Oscillations and hippocampal-prefrontal synchrony. Curr Opin Neurobiol 21, 486491, doi:10.1016/j.conb.2011.02.012 (2011).

38 Jones, M. W. \& Wilson, M. A. Theta rhythms coordinate hippocampal-prefrontal interactions in a spatial memory task. PLoS.Biol. 3, e402 (2005).

39 Buzsaki, G. \& Draguhn, A. Neuronal oscillations in cortical networks. Science. 304, 1926-1929 (2004).

40 Benchenane, K., Tiesinga, P. H. \& Battaglia, F. P. Oscillations in the prefrontal cortex: a gateway to memory and attention. Curr Opin Neurobio/ 21, 475-485, doi:10.1016/j.conb.2011.01.004 (2011).

41 Hyman, J. M., Zilli, E. A., Paley, A. M. \& Hasselmo, M. E. Working Memory Performance Correlates with Prefrontal-Hippocampal Theta Interactions but not with Prefrontal Neuron Firing Rates. Front Integr Neurosci 4, 2, doi:10.3389/neuro.07.002.2010 (2010).

42 Benchenane, K. et al. Coherent theta oscillations and reorganization of spike timing in the hippocampal-prefrontal network upon learning. Neuron 66, 921-936, doi:10.1016/j.neuron.2010.05.013 (2010).

43 Abraham, N. M. et al. Maintaining accuracy at the expense of speed: Stimulus similarity defines odor discrimination time in mice. Neuron 44, 865-876 (2004).

44 Rinberg, D., Koulakov, A. \& Gelperin, A. Speed-accuracy tradeoff in olfaction. Neuron 51, $351-$ 358, doi:10.1016/j.neuron.2006.07.013 (2006). 
104245 Uchida, N. \& Mainen, Z. F. Speed and accuracy of olfactory discrimination in the rat. Nat

$1043 \quad$ Neurosci 6, 1224-1229, doi:10.1038/nn1142 (2003).

104446 Eichenbaum, H. Using olfaction to study memory. Ann N Y Acad Sci 855, 657-669 (1998).

$104547 \quad$ Frederick, D. E. et al. Gamma and Beta Oscillations Define a Sequence of Neurocognitive Modes 1046 Present in Odor Processing. J Neurosci 36, 7750-7767 (2016).

1047

1048

1049

1050

1051

$1052 \quad 50$

1053

1054

1055

1056

1057

1058

1059

1060

1061

1062

1063

1064

1065

1066

1067

1068

1069

1070

1071

1072

1073

1074

1075

$48 \quad$ Kay, L. M. \& Beshel, J. A beta oscillation network in the rat olfactory system during a 2alternative choice odor discrimination task. J Neurophysiol 104, 829-839, doi:10.1152/jn.00166.2010 (2010).

49 Rangel, L. M. et al. Rhythmic coordination of hippocampal neurons during associative memory processing. Elife 5, doi:10.7554/eLife.09849 (2016).

50 Stopfer, M., Jayaraman, V. \& Laurent, G. Intensity versus identity coding in an olfactory system. Neuron 39, 991-1004 (2003).

51 Nguyen Chi, V. et al. Hippocampal Respiration-Driven Rhythm Distinct from Theta Oscillations in Awake Mice. J Neurosci 36, 162-177 (2016).

52 Kepecs, A., Uchida, N. \& Mainen, Z. F. The Sniff as a Unit of Olfactory Processing. Chemical Senses 31, 167-179 (2006).

53 Lockmann, A. L. V., Laplagne, D. A., Leão, R. N. \& Tort, A. B. L. A Respiration-Coupled Rhythm in the Rat Hippocampus Independent of Theta and Slow Oscillations. J Neurosci 36, 5338-5352 (2016).

54 Verhagen, J. V., Wesson, D. W., Netoff, T. I., White, J. a. \& Wachowiak, M. Sniffing controls an adaptive filter of sensory input to the olfactory bulb. Nature Neuroscience 10, 631-639 (2007).

55 Kay, L. M. Theta oscillations and sensorimotor performance. Proc. Natl. Acad. Sci. U.S.A 102, 3863-3868 (2005).

56 Fujisawa, S., Amarasingham, A., Harrison, M. T. \& Buzsaki, G. Behavior-dependent short-term assembly dynamics in the medial prefrontal cortex. Nat Neurosci 11, 823-833, doi:10.1038/nn.2134 (2008).

57 Fujisawa, S. \& Buzsaki, G. A 4 Hz oscillation adaptively synchronizes prefrontal, VTA, and hippocampal activities. Neuron 72, 153-165, doi:S0896-6273(11)00739-2 [pii] 10.1016/j.neuron.2011.08.018 (2011).

58 Martin, C., Beshel, J. \& Kay, L. M. An olfacto-hippocampal network is dynamically involved in odor-discrimination learning. J Neurophysio/ 98, 2196-2205, doi:10.1152/jn.00524.2007 (2007).

Alvarez, P., Wendelken, L. \& Eichenbaum, H. Hippocampal formation lesions impair performance in an odor-odor association task independently of spatial context. Neurobiol. Learn. Mem. 78, 470-476 (2002). 
$1076 \quad 60$

1077

$1078 \quad 61$

1079

1080

1081

1082

1083

1084

1085

1086

1087

1088

1089

1090

$1091 \quad 66$

1092

1093

1094

1095

1096

1097

1098

1099

1100

1101

1102

1103

1104

1105

1106

1107

1108

1109

Peters, G. J., David, C. N., Marcus, M. D. \& Smith, D. M. The medial prefrontal cortex is critical for memory retrieval and resolving interference. Learning \& Memory 20, 201-209 (2013).

61 Otto, T. \& Eichenbaum, H. Complementary roles of the orbital prefrontal cortex and the perirhinal- entorhinal cortices in an odor-guided delayed-nonmatching-to-sample task. Behav Neurosci 106, 762-775 (1992).

62 Eichenbaum, H., Fagan, A. \& Cohen, N. J. Normal olfactory discrimination learning set and facilitation of reversal learning after medial-temporal damage in rats: implications for an account of preserved learning abilities in amnesia. J Neurosci 6, 1876-1884 (1986).

63 Moser, M. B. \& Moser, E. I. Distributed encoding and retrieval of spatial memory in the hippocampus. J Neurosci 18, 7535--7542 (1998).

64 Wiltgen, B. J. et al. The hippocampus plays a selective role in the retrieval of detailed context memories. Curr Biol 20, 1336-1344 (2010).

65 Churchwell, J. C., Morris, A. M., Musso, N. D. \& Kesner, R. P. Prefrontal and hippocampal contributions to encoding and retrieval of spatial memory. Neurobiol Learn Mem 93, 415-421, doi:10.1016/j.nlm.2009.12.008 (2010).

66 Kay, L. M. in Progress in Brain Research Vol. 208 (ed Donald A. Wilson Edi Barkai) (Elsevier, 2014).

67 Kay, L. M. et al. Olfactory oscillations: the what, how and what for. Trends in Neurosciences 32, 207-214 (2009).

68 Neville, K. R. \& Haberly, L. B. Beta and Gamma Oscillations in the Olfactory System of the Urethane-Anesthetized Rat. Journal of Neurophysiology 90, 3921-3930 (2003).

69 Martin, C., Gervais, R., Messaoudi, B. \& Ravel, N. Learning-induced oscillatory activities correlated to odour recognition: a network activity. European Journal of Neuroscience 23, 18011810 (2006).

70 Donoghue, J. P., Sanes, J. N., Hatsopoulos, N. G. \& Gaál, G. Neural discharge and local field potential oscillations in primate motor cortex during voluntary movements. Journal of Neurophysiology 79, 159-173 (1998).

71 Sanes, J. N. \& Donoghue, J. P. Oscillations in local field potentials of the primate motor cortex during voluntary movement. Proc Natl Acad Sci U S A 90, 4470-4474 (1993).

72 Carr, M. F., Jadhav, S. P. \& Frank, L. M. Hippocampal replay in the awake state: a potential substrate for memory consolidation and retrieval. Nat Neurosci 14, 147-153, doi:10.1038/nn.2732 (2011).

73 Joo, H. R. \& Frank, L. M. The hippocampal sharp wave-ripple in memory retrieval for immediate use and consolidation. Nat Rev Neurosci 19, 744-757, doi:10.1038/s41583-018-0077-1 (2018). 
$111074 \quad$ Norman, Y. et al. Hippocampal sharp-wave ripples linked to visual episodic recollection in humans. Science 365, doi:10.1126/science.aax1030 (2019).

111275 Buzsaki, G., Leung, L. W. \& Vanderwolf, C. H. Cellular bases of hippocampal EEG in the behaving rat. Brain Res. 287, 139-171 (1983).

1114

1115

1116

76 Schoenbaum, G. \& Eichenbaum, H. Information coding in the rodent prefrontal cortex. I. Singleneuron activity in orbitofrontal cortex compared with that in pyriform cortex. J. Neurophysiol. 74, 733-750 (1995).

77 Taxidis, J. et al. Differential Emergence and Stability of Sensory and Temporal Representations in Context-Specific Hippocampal Sequences. Neuron 108, 1-15 (2020).

78 Jadhav, S. P., Rothschild, G., Roumis, D. K. \& Frank, L. M. Coordinated excitation and inhibition of prefrontal ensembles during awake hippocampal sharp-wave ripple events. Neuron 90, 113-127, doi:10.1016/j.neuron.2016.02.010 (2016).

79 Siapas, A. G., Lubenov, E. V. \& Wilson, M. A. Prefrontal phase locking to hippocampal theta oscillations. Neuron 46, 141-151, doi:10.1016/j.neuron.2005.02.028 (2005).

80 Tort, A. B., Komorowski, R. W., Manns, J. R., Kopell, N. J. \& Eichenbaum, H. Theta-gamma coupling increases during the learning of item-context associations. Proc Natl Acad Sci U S A 106, 20942-20947, doi:0911331106 [pii]10.1073/pnas.0911331106 (2009).

81 Hermer-Vazquez, R., Hermer-Vazquez, L., Srinivasan, S. \& Chapin, J. K. Beta- and gammafrequency coupling between olfactory and motor brain regions prior to skilled, olfactory-driven reaching. Experimental Brain Research 180, 217-235 (2007).

82 Buzsáki, G. Hippocampal sharp wave-ripple: A cognitive biomarker for episodic memory and planning. Hippocampus 25, 1073-1188, doi:10.1002/hipo.22488 (2015).

83 Beshel, J., Kopell, N. \& Kay, L. M. Olfactory bulb gamma oscillations are enhanced with task demands. J Neurosci 27, 8358-8365, doi:10.1523/JNEUROSCI.1199-07.2007 (2007).

84 Martin, C. \& Ravel, N. Beta and gamma oscillatory activities associated with olfactory memory tasks: different rhythms for different functional networks? Frontiers in Behavioral Neuroscience 8 (2014).

85 Eichenbaum, H., Kuperstein, M., Fagan, A. \& Nagode, J. Cue-sampling and goal-approach correlates of hippocampal unit activity in rats performing an odor-discrimination task. J Neurosci 7, 716-732 (1987).

86 Terada, S., Sakurai, Y., Nakahara, H. \& Fujisawa, S. Temporal and Rate Coding for Discrete Event Sequences in the Hippocampus. Neuron 94, 1248-1262 (2017).

87 Komorowski, R. W., Manns, J. R. \& Eichenbaum, H. Robust conjunctive item-place coding by hippocampal neurons parallels learning what happens where. J Neurosci 29, 9918-9929 (2009). 
$114488 \quad$ Ferbinteanu, J., Shirvalkar, P. \& Shapiro, M. L. Memory modulates journey-dependent coding in the rat hippocampus. J Neurosci 31, 9135-9146, doi:10.1523/JNEUROSCI.1241-11.2011 (2011).

114689 Liu, X. et al. Optogenetic stimulation of a hippocampal engram activates fear memory recall. Nature 484, 381-385, doi:10.1038/nature11028 (2012).

114890 DeNardo, L. A. et al. Temporal evolution of cortical ensembles promoting remote memory retrieval. Nat Neurosci 22, 460-469 (2019). hippocampal sharp-wave-ripple events and vicarious trial and error behaviors in decision making. Neuron 92, 975-982, doi:10.1016/j.neuron.2016.10.028 (2016).

Zhong, W. et al. Selective entrainment of gamma subbands by different slow network oscillations. Proc Natl Acad Sci U S A 114, 4519-4524 (2017). support spatial memory. Science 336, 1454-1458, doi:10.1126/science.1217230 (2012). doi:10.1523/JNEUROSCI.2291-17.2017 (2017). between neuronal oscillations of different frequencies. J Neurophysiol 104, 1195-1210, doi:jn.00106.2010 [pii] 10.1152/jn.00106.2010 (2010).

1175102 Karlsson, M. P. \& Frank, L. M. Awake replay of remote experiences in the hippocampus. Nat. 
bioRxiv preprint doi: https://doi.org/10.1101/2020.06.08.140939; this version posted February 5, 2021. The copyright holder for this preprint (which was not certified by peer review) is the author/funder, who has granted bioRxiv a license to display the preprint in perpetuity. It is made available under aCC-BY-NC-ND 4.0 International license.

1177103 Skaggs, W. E., McNaughton, B. L., Gothard, K. \& Markus, E. in Advanced in Neural Information Processing Systems (eds S. Hanson, J. D. Cowan, \& C. L. Giles) 1030-1037 (Morgan Kaufmann Publishers, 1993). 


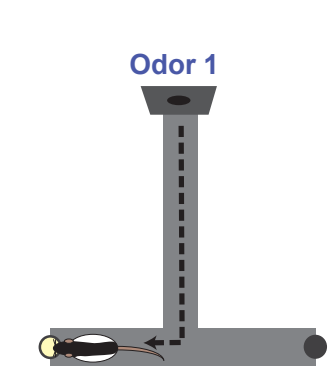

Reward 1

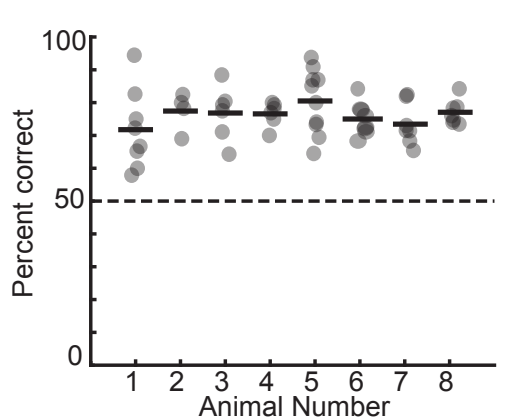

d

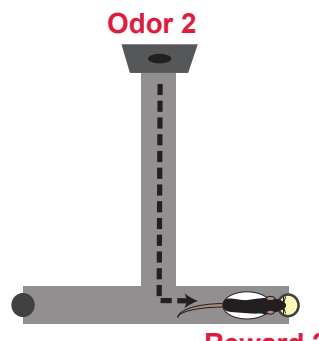

Reward 2

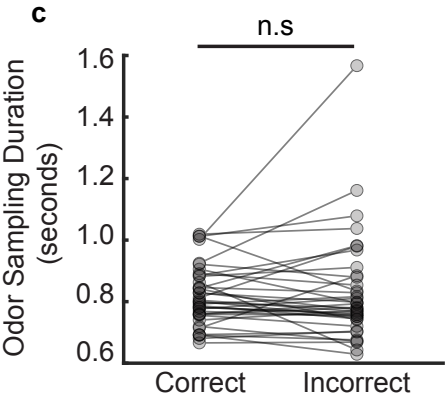



Reward

$98 \%$

Same Direction

Correct

Trials

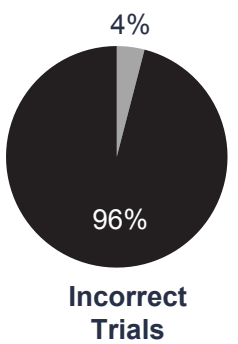

Reward

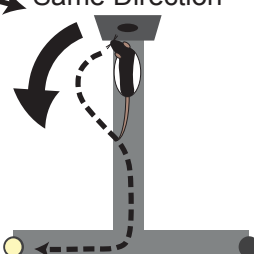


1181 a. Schematic of the odor-cued T-maze task. Odors 1 (heptanol) and 2 (ethyl butyrate) were 1182 delivered at the odor port in pseudo-random order. Presentation of Odors 1 and 2 was 1183 associated with milk reward at Reward locations 1 and 2, respectively. Animals had to recall 1184 odor-space associations on each trial and utilize the association to choose the correct 1185 reward location.

1186 b. Performance of each animal $(n=8)$ on the odor-cued T-maze task (animals $1-5)$ or 1187 truncated odor-cued task (animals 6 - 8; truncated task, without spatial delay period) across 1188 multiple sessions (grey dots). Animal average is indicated by black bars. Dashed line 1189 indicates chance level.

1190 c. Odor sampling duration across all sessions $(n=38)$ on correct and incorrect trials (signed1191 rank test, $\mathrm{p}=0.71)$.

1192 d. Turn direction away from odor port in relation to chosen reward well. Pie charts indicate the 1193 fraction of trials in which, at the odor port, animals turned in the same direction versus the 1194 opposite direction as the reward well that they would ultimately choose. Sessions in which 1195 animals ran the truncated task without spatial delay period were excluded. (correct trials: $n=$ 11961624 same direction, 32 opposite direction, binomial test, $\mathrm{p}=0$; incorrect trials: $\mathrm{n}=499$ 1197 same direction, 21 opposite direction, $p=1.4 \mathrm{e}-108)$. 
a

Thermocouple (TC)

-400UViv Win

Hippocampus (CA1)

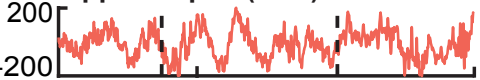

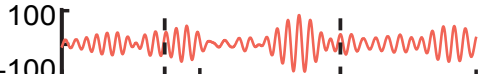

3
3
$\frac{0}{2}$
$\frac{0}{2}$
$\frac{1}{2}$
$\frac{0}{2}$
$\frac{0}{2}$

Prefrontal Cortex (PFC)

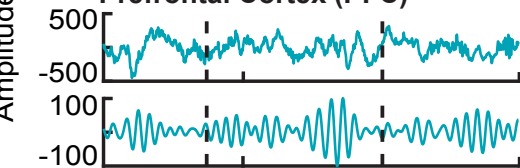

Olfactory Bulb (OB)
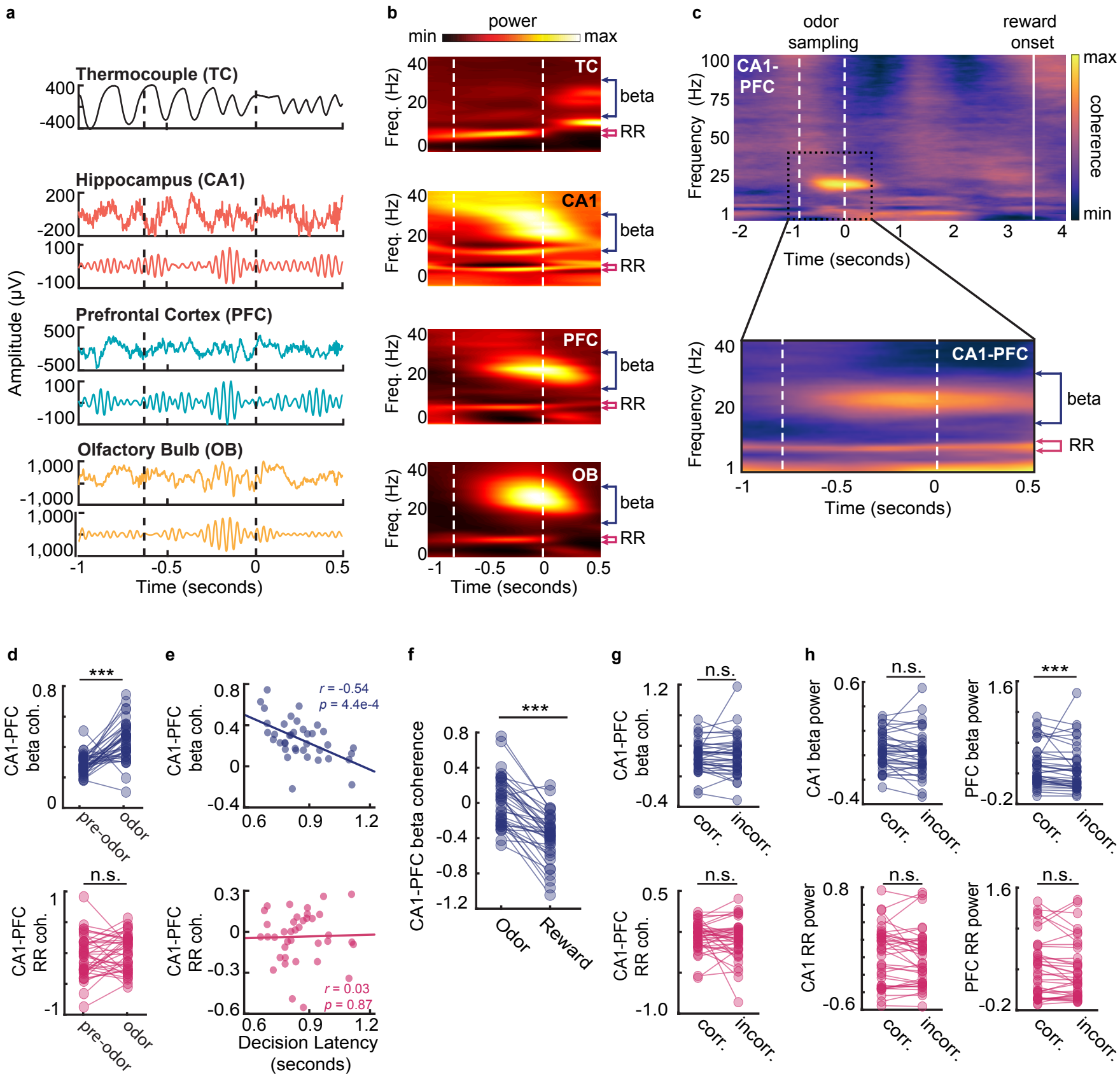

g

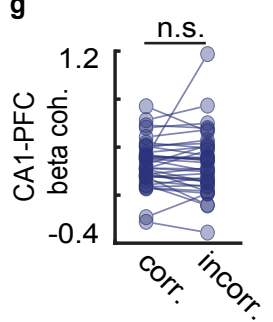

h
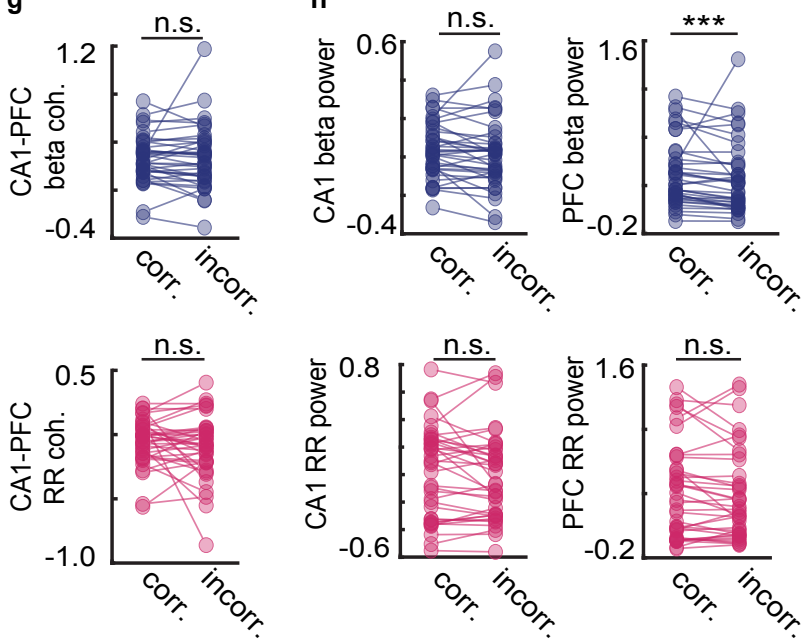



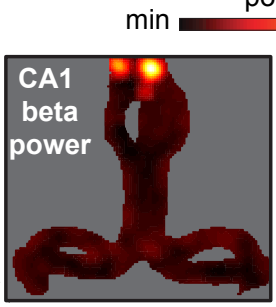

$\max$
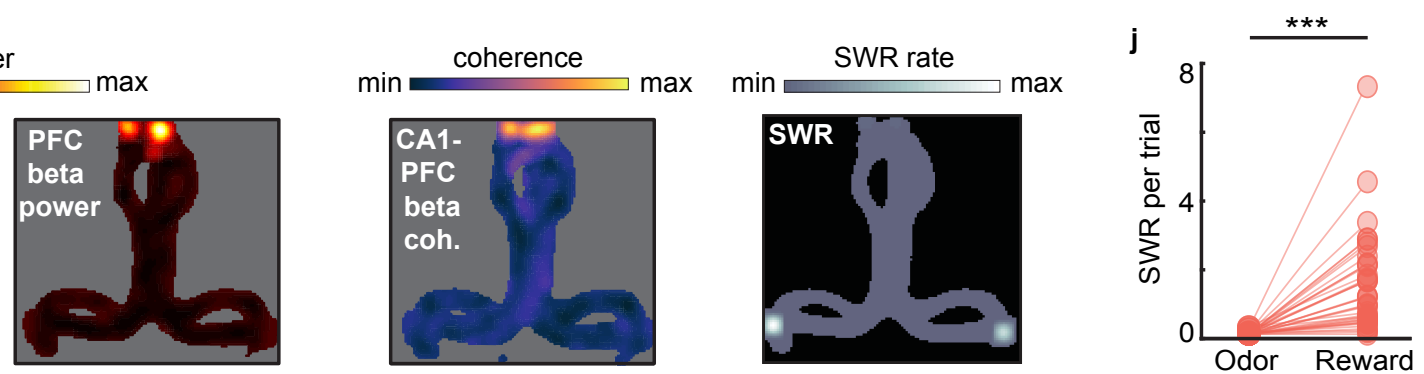

$15 \mathrm{~cm}$ 
a. Examples of thermocouple and LFP traces from one tetrode in each region during presentation of odor from one trial, aligned to odor port disengagement. Area between dashed lines indicates odor sampling period. Top to bottom: Respiratory rhythm recorded via thermocouple, CA1 signal, beta band $(15-30 \mathrm{~Hz})$ filtered CA1 signal, PFC signal, beta band filtered PFC signal, OB signal, beta band filtered OB signal.

b. Time-frequency plot showing power spectra aligned to odor offset. Color scale represents z-scored power. Area between dashed lines indicates average odor sampling period. Beta band is marked by blue bracket $(15-30 \mathrm{~Hz})$. RR band is marked by pink bracket $(7-8 \mathrm{~Hz})$. Thermocouple signal (TC), $\mathrm{n}=12$ sessions, $\max 0.69$, $\min -0.31$; CA1: $n=38$ sessions, $\max 0.26, \min -0.35 ;$ PFC: $n=38$ sessions, $\max 0.60, \min -0.16$; and OB: $\mathrm{n}=38$ sessions, $\max 2.17$, $\min -0.24$.

c. Top: CA1-PFC coherence spectra for one animal during the full task time window from odor sampling (area between white dashed lines, aligned to odor offset) to average reward onset time (solid line) ( $n=4$ sessions, max 0.84; min -0.41). Bottom: CA1-PFC coherence aligned to odor offset across all animals $(n=38$ sessions, max 0.51 ; min 0.34). Color scale represents z-scored power. pre-odor periods. (signed-rank tests, $n=38$ sessions. Beta coherence: $p=6.7 e-6^{* * *} ; R R$ coherence: $p=0.36)$. correct trials. $\left(n=38\right.$ sessions, beta coherence: $r=-0.54, p=4.4 e-4^{* * *}$; RR coherence: $r$ $=0.03, p=0.87$.

f. CA1-PFC beta coherence during $500 \mathrm{~ms}$ window preceding odor port exit, and $500 \mathrm{~ms}$ window preceding reward well exit (signed rank test, $n=38$ sessions, $p=1.2 e-7^{\star * *}$ ). 
g. Beta (top) and RR (bottom) CA1-PFC coherence on correct and incorrect trials. (Signedrank tests, $n=38$ sessions. Beta coherence: $p=0.38 ; R R$ coherence: $p=0.81)$.

h. Beta (top row) and respiratory rhythm (RR) (bottom row) power on correct and incorrect trials in CA1 and PFC. (Signed-rank tests. $n=38$ sessions. Beta power: CA1: $p=0.08$; PFC: $p=8.3 e-4^{* * *}$. RR power: CA1: $p=0.26 ;$ PFC: $\left.p=0.16.\right)$

i. Maps of T-maze showing the spatial distribution of z-scored CA1 beta power (left. max 1.49; min -0.19), PFC beta power (middle-left, max 1.63; min -0.18), CA1-PFC beta coherence (middle-right, max 1.16; min -0.19), and SWR rate (right, max 1.34; min 0). Plots are for a single session from one animal. correct trials (signed-rank test, $n=38$ sessions, $p=1.1 e-7^{\star * *}$ ). 
a

CA1

interactive cells: 937

119

pyramidal cells: 818

taskunresponsive: 693

taskresponsive:

125

non-selective: 42

PFC

interactive cells: 508

64

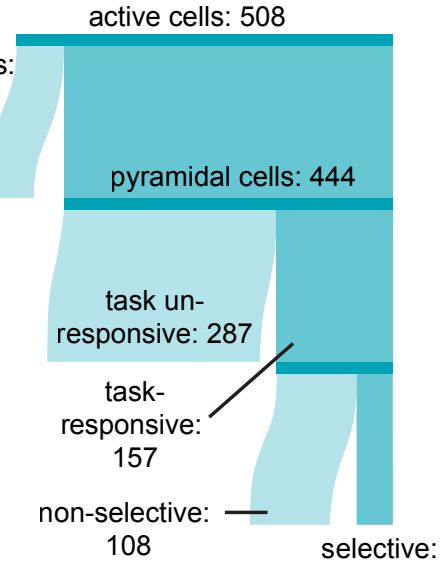

49 b
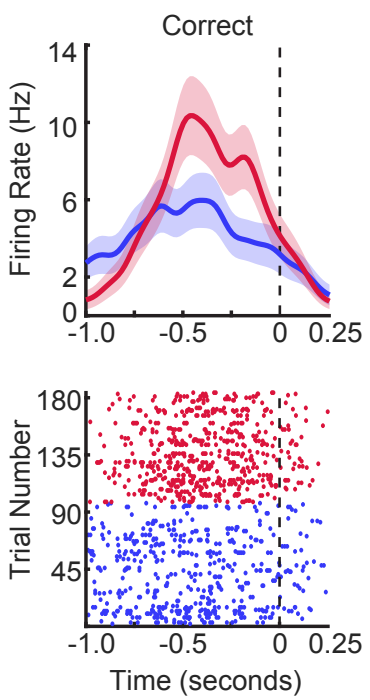

g

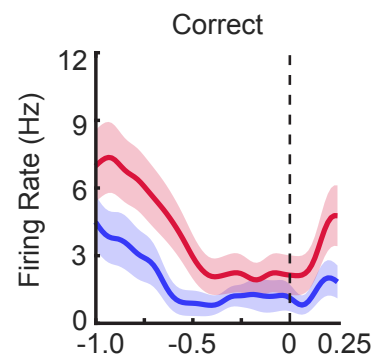

h

- Odor 1 response
- Odor 2 response
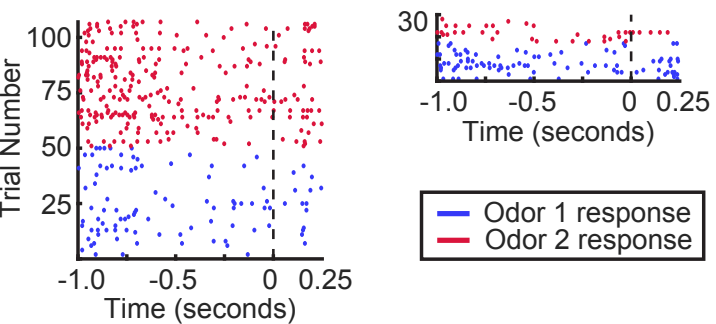

d


i
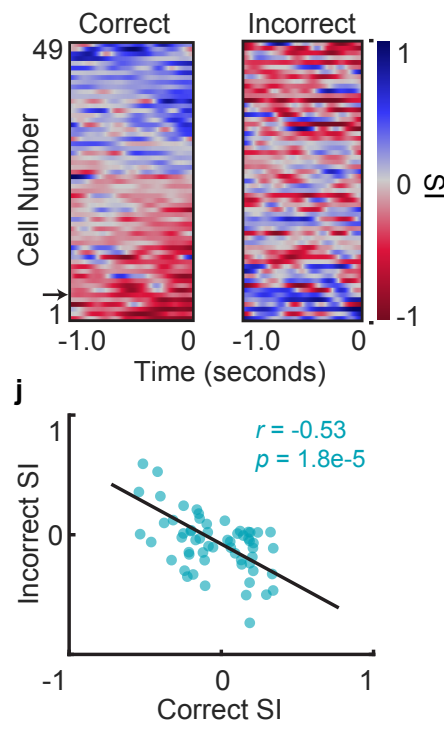
a. Sankey diagram showing the number of CA1 cells that were classified into different categories. Sizes of partitions are proportional to raw numbers.

b. Example PSTH and raster plot for a single choice-selective CA1 neuron on correct trials, aligned to odor-port disengagement. Shaded areas indicate s.e.m. Firing rates are shown in $\mathrm{Hz}$, referring to spikes/second.

c. PSTH and raster plot for the same neuron in (b), but only for incorrect trials.

d. Selectivity index (SI) of all choice-selective cells in CA1 on correct trials (left) and incorrect trials (right), aligned to odor-port disengagement. SI is calculated as the difference in firing rate between Odor 1 trials and Odor 2 trials, divided by the sum of these firing rates. $\mathrm{SI}$ is color coded, where blue indicates an SI of 1 (absolute Choice 1 preference), red indicates an SI of -1 (absolute Choice 2 preference) and grey indicates an SI of 0 (not selective). Cells are sorted according to peak selectivity on correct trials and sorting order is the same for both plots. $\left.=42, r=-0.42, p=0.003^{* *}\right)$

f-j. Same as a-e, but for PFC cells. Correlation in $\mathrm{j}: \mathrm{n}=49, \mathrm{r}=-0.53, \mathrm{p}=1.8 \mathrm{e}-5^{\star \star *}$. 



f


g
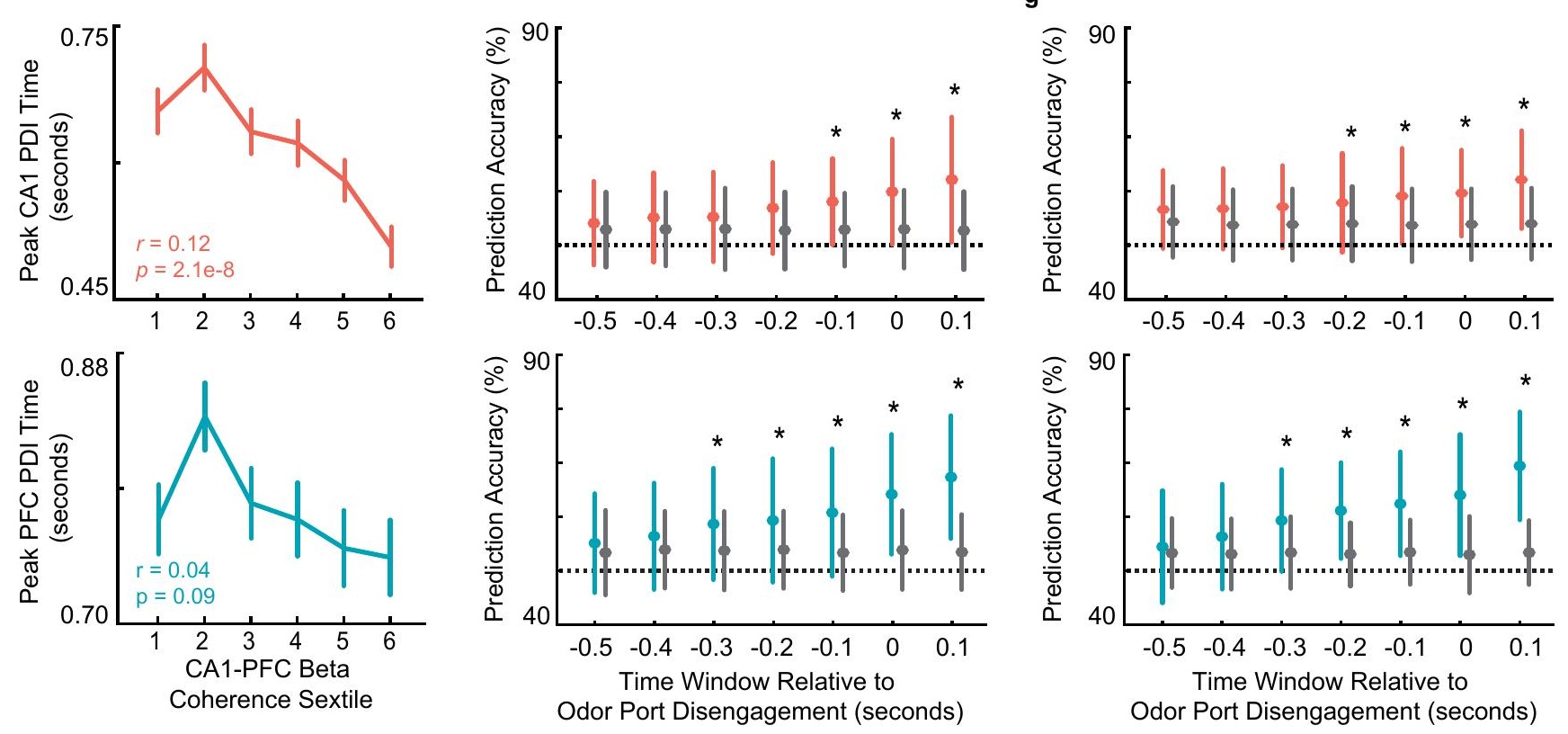

h
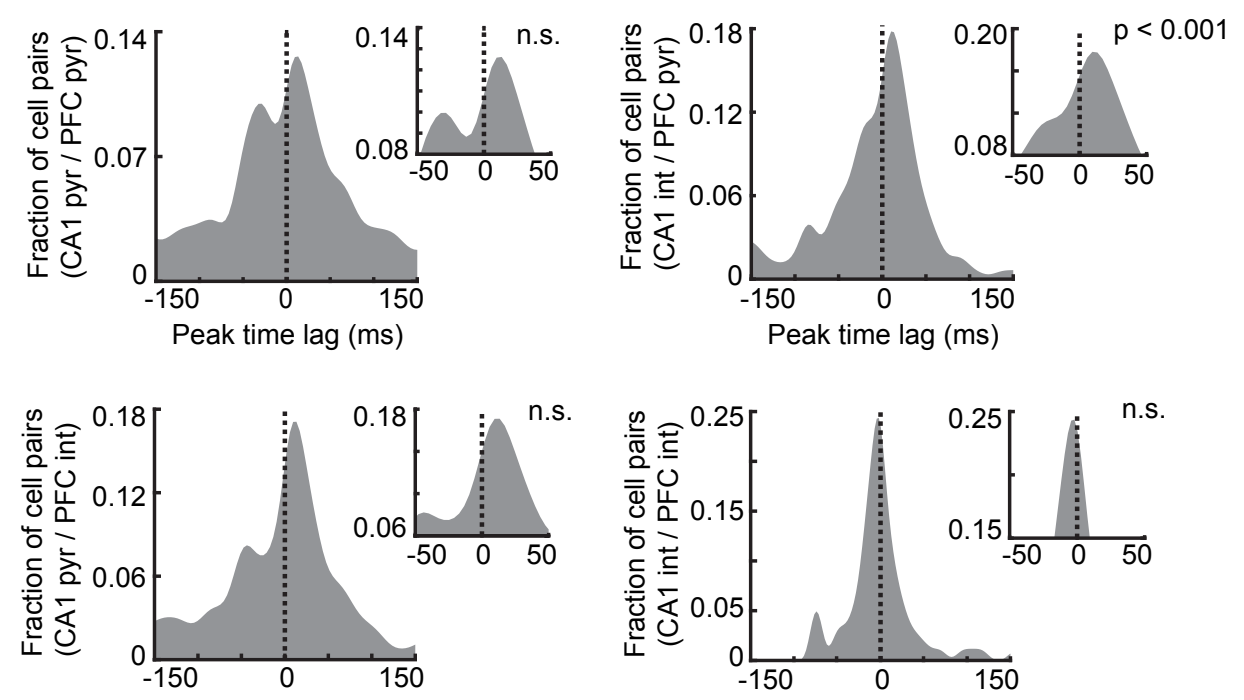

Peak time lag (ms) 
Figure 4: Neural ensemble responses during decision making are governed by beta coordination and predict the upcoming choice

1260

1261

1262

1263

1264

1265

1266

1267

1268

1269

1270

1271

1272

1273

1274

1275

1276

1277

1278

1279

1280

1281

a. Normalized firing rate of all task-responsive cells in CA1 (top) and PFC (bottom) during a 1 second window before odor port disengagement.

b. Discriminability between population vectors (PVs) on Odor 1 versus Odor 2 presentations, for CA1 task-responsive population (top, $n=125$ cells) and PFC task-responsive population (bottom, $\mathrm{n}=157$ cells), across time bins aligned to odor port disengagement. Dissimilarity between PVs is scaled and shown as a PV differential index (PDI), where 0 indicates identical responses. Colored line indicates real data, grey shaded area indicates $95 \%$ confidence intervals of shuffled distribution.

c. PV divergence time in CA1 and PFC. Divergence time is quantified as the first time point where the real data PDI surpasses the mean +2 s.d. of the shuffled PDI distribution. Bootstrap test, $\mathrm{n}=50$ samples with replacement, 500 iterations. Boxplots indicate interquartile ranges of bootstrapped distributions. CA1: median $=0.19$ seconds prior to odor-port disengagement, interquartile range $=0.06$; PFC: median $=0.23$ seconds prior to odor-port disengagement, interquartile range $=0.06$; (bootstrap test; $p=0.31$ )

d. Timing of peak PV discriminability (PDI) on each trial aligned to odor onset as a function of decision latency for CA1 (top) and PFC (bottom). Decision latencies were divided into six equally sized subgroups (sextiles) for visualization, but correlation coefficients were calculated using trials. Error bars show s.e.m. at each sextile (CA1: $n=2071$ trials, $r=$ $0.16, p=1.1 e-12^{* * *} ;$ PFC: $n=2081$ trials, $\left.r=r=0.06, p=0.007^{* *}\right)$. Individual trial rasters in Supplementary Figure 4j.

e. Timing of peak PV discriminability (PDI) on each trial as a function of beta coherence. Beta coherence distribution was divided into six equally sized subgroups (sextiles) for 
visualization, but correlation coefficients were calculated using trials. Error bars show s.e.m. at each sextile (CA1: $r=-0.12, p=2.1 e-8^{* * *} ;$ PFC: $\left.r=-0.04, p=0.09\right)$.

1284 f. Prediction of animal's choice of reward well by a GLM according to ensemble activity during odor sampling across varying length time windows aligned to decision time. Colored

g. As in (i) but including both putative pyramidal and interneurons in ensembles for GLM prediction.

h. Histograms showing distributions of peak timings of cross-correlations between CA1 and PFC cell pairs. Peaks falling above zero indicate CA1 leading, whereas peaks falling below zero indicate PFC leading. One-sample Wilcoxson signed rank tests $\left(\mathrm{H}_{0}: \mu=0 \mathrm{~ms}\right)$. Topleft: CA1-PFC pyramidal pairs, $n=603$ pairs, $p=0.07$; top-right: CA1 interneuron-PFC pyramidal pairs, $n=462$ pairs, $p=6.0 e-4^{* * *}$; bottom-left: CA1 pyramidal-PFC interneuron pairs, $n=130$ pairs, $p=0.14$, bottom right: CA1-PFC interneuron pairs, $n=85$ pairs, $p=$ 
a. Example spike-phase histograms from two example cells that are phase locked to the beta rhythm. Top: CA1 cell (Rayleigh $Z$ test, $n=242$ spikes, $z=15.5, p=1.5 e-7$ ); bottom: PFC cell $(n=99$ spikes, $z=4.86, p=0.007)$. Phase axes are duplicated for visibility.

b. Polar histogram of preferred beta phases for all task-responsive CA1 ( $n=125$ cells, Rayleigh $Z$ test, $z=7.9, p=3.4 e-4)$ and PFC ( $n=157$ cells, $z=5.0, p=0.006)$ cells. Arrows

c. Rayleigh Z scores of spike-phases for all task-responsive pyramidal cells phase-locked to the beta rhythm locally and cross-regionally on correct versus incorrect trials. (signed-rank tests, CA1 cells phase locked to CA1 beta: $n=28$ cells, $p=0.03^{*} ;$ PFC-PFC: $n=8$ cells, $p=$

d. As in (c), but for spike-phases for all pyramidal cells phase-locked to local RR. (signed-rank tests, CA1-CA1: $n=103$ cells, $p=0.04^{*}$; PFC-PFC: $n=21$ cells, $p=0.24 ;$ CA1-PFC: $n=85$

e. Percentage of cells in CA1 and PFC that were phase locked to either beta or RR (z-tests for cells, $p=0.27 ;$ PFC-CA1: $n=49$ cells, $p=0.30$ ).

f. Percentage of cells that are choice-selective (solid bars), phase-locked to the beta rhythm (outlined bars), and both choice-selective and phase-locked (striped bars). Grey bars proportions; CA1: $n=37$ cells phase-locked to beta, $n=112$ cell phase-locked to $R R, z=-$ 9.8, $p=3.2 e-20 ;$ PFC: $n=17$ cells phase-locked to beta, $n=60$ cells phase-locked to $R R, z$ $=-5.5, p=1.8 e-8)$. indicate chance level of cells being both choice-selective and phase-locked, error bars indicate the range of cell percentages that fall within the $95 \%$ bounds of the chance binomial distribution. (binomial test, CA1: $p=0.08$; PFC: $p=0.16$ ). 
1326 g. Polar histogram of preferred beta phases for all task-responsive CA1 interneurons phase-

1327 locked to the local CA1 beta rhythm (top, $n=44$ cells, $z=8.0, p=2.9 e-4^{* * *}$ ) and CA1

1328 interneurons phase-locked to the PFC beta rhythm (bottom, $n=25$ cells, $z=22.5, p=1.8 e-$

$132911^{* * *}$ ) Arrows indicate mean phase direction and magnitude.

1330 h. Rayleigh Z scores of spike-phases for all task-responsive CA1 interneurons phase-locked

1331 locally and cross-regionally to the beta rhythm on correct versus incorrect trials. (signed-rank

1332 tests, CA1-CA1: $n=44$ cells, $p=8.2 e-9^{\star * *} ;$ CA1-PFC: $n=25$ cells, $\left.p=1.2 e-5^{\star * *}\right)$.

1333 i. As in (f), but for interneurons. (binomial test; CA1: $p=0.11$; PFC: $p=0.20$ ) 

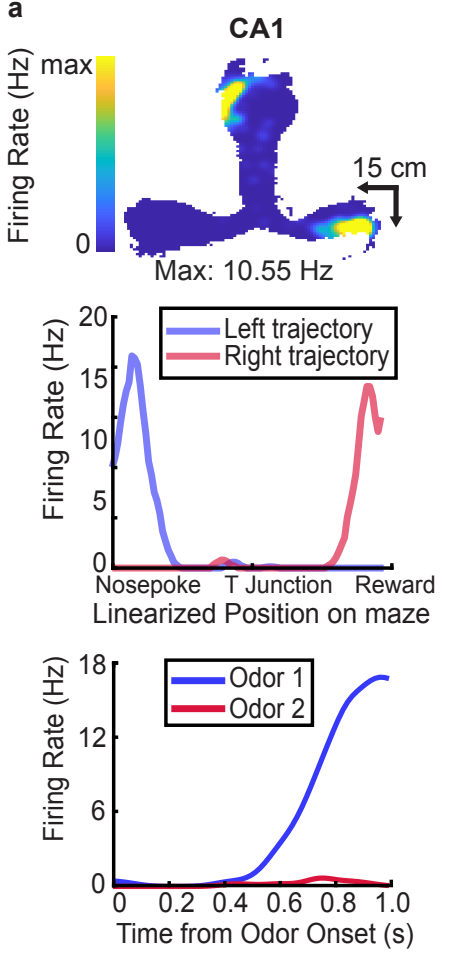
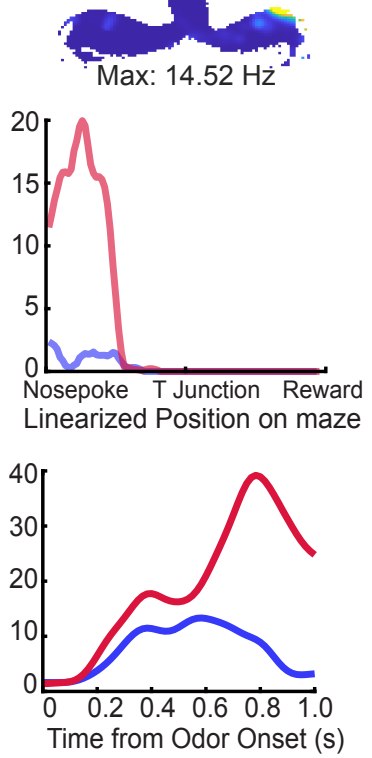

"Max: $10.54 \mathrm{~Hz}$
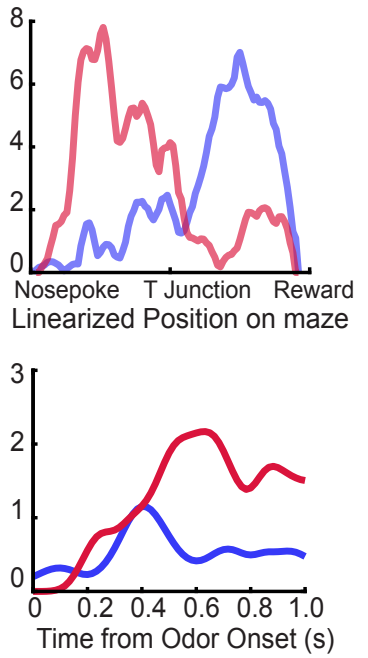

C


Max: $6.04 \mathrm{~Hz}^{-}$
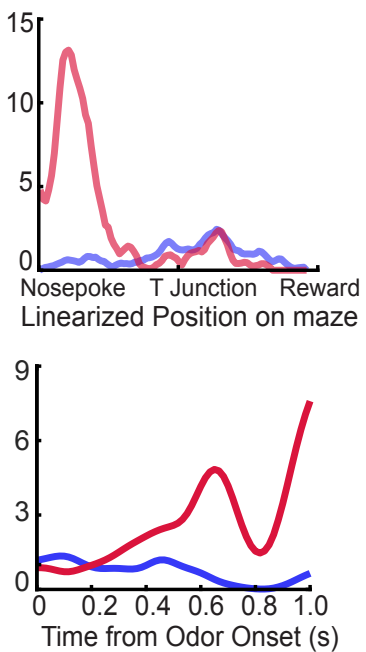

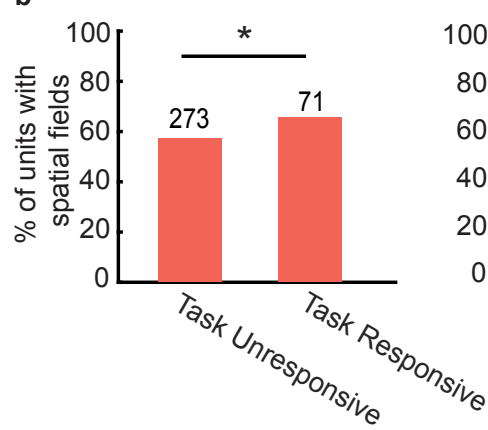

d

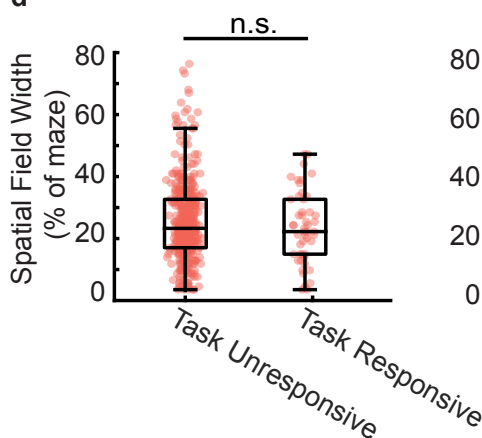

f

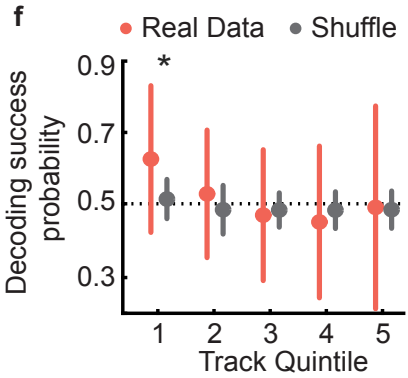

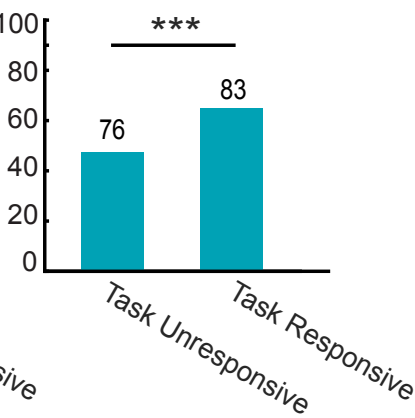

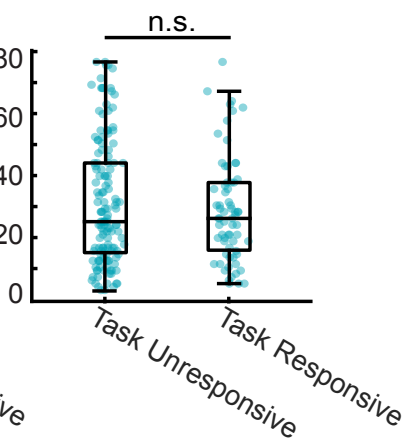

e

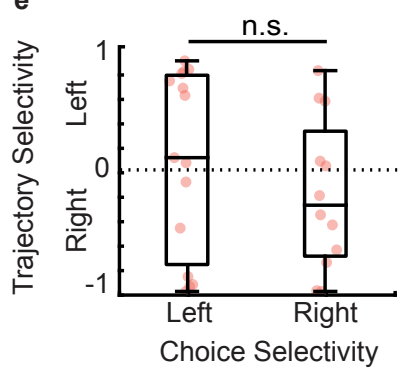

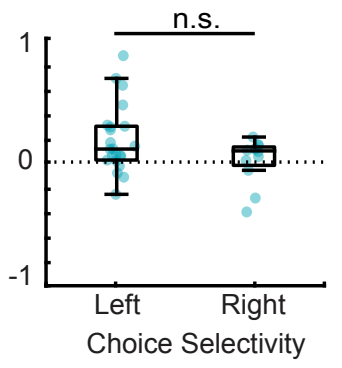
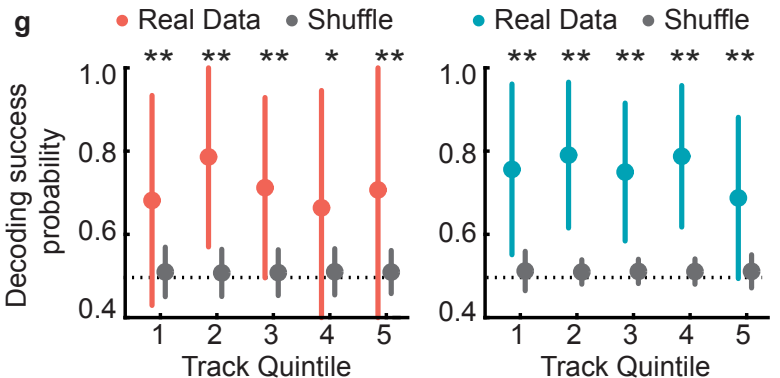
a. Example choice-selective units (CA1, first two columns, PFC, last two columns) with spatial fields on the track. Top row: Heat map illustrating spatial fields during run bouts. Middle row: Linearized spatial tuning curves for outbound left and right run trajectories. Bottom row:

b. Spatial field prevalence in task-unresponsive and task-responsive cells in CA1 (left) and PFC (right). CA1: 273/477 (57\%) of task-unresponsive cells, and 71/108 (66\%) of taskresponsive cells had spatial fields (binomial test: $\left.p=0.03^{*}\right)$; PFC: 76/160 (48\%) of taskunresponsive cells, and $83 / 128(66 \%)$ of task-responsive cells had spatial fields (binomial

c. Correlation between firing rates during run and firing rates during odor sampling period for all putative pyramidal cells in CA1 (left) and PFC (right). (CA1: $n=585$ cells, $r=0.39, p=$ 3.0e-37; PFC: $n=288$ cells, $r=0.68, p=1.2 e-62)$.

d. Spatial field width for odor unresponsive and odor responsive units in CA1 (left) and PFC (right). If a unit had a spatial field on both outbound trajectories, each field was counted separately. (Rank-sum tests; CA1: $n=452$ fields, $p=0.15$; PFC: $n=207$ fields, $p=0.92$ ). sum tests, CA1: $p=0.26$; PFC: $p=0.20)$.

f. Decoding of choice identity by naïve Bayesian classifier according to CA1 (left) and PFC (right) task-responsive ensemble activity at five equally sized spatial quintiles along the full run trajectory of the maze. 1 spatial quintile $=24.6 \mathrm{~cm}(123 \mathrm{~cm} / 5)$. Colored error bars indicate real data mean \pm s.d. whereas grey error bars indicate shuffled data mean \pm s.d. 
1359 Stars indicate quintiles where the fraction of correctly decoded trials was significantly higher

1360 than the fraction from the shuffled data (rank-sum tests, ${ }^{*}=p<0.05$ ).

1361 g. As in (f), but decoding of choice identity according to CA1 (left) and PFC (right) spatially-

1362 modulated ensemble activity at each spatial quintile. (rank-sum tests, ${ }^{*}=p<0.05,{ }^{* *}=p<$

$1363 \quad 0.01)$ 